\title{
Extending two-Higgs-doublet models by a singlet scalar field - The case for dark matter
}

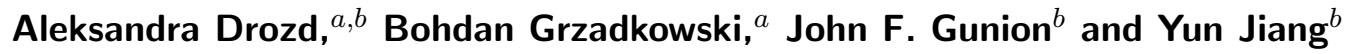 \\ ${ }^{a}$ Faculty of Physics, University of Warsaw, \\ 00-681 Warsaw, Poland \\ ${ }^{b}$ Department of Physics, University of California, \\ Davis, CA 95616, U.S.A. \\ E-mail: aleksandra.drozd@fuw.edu.pl, bohdan.grzadkowski@fuw.edu.pl, \\ jfgunion@ucdavis.edu, yunjiang@ucdavis.edu
}

ABSTRACT: We extend the two-Higgs doublet models of Type I and Type II by adding a real gauge-singlet scalar $S$ dark matter candidate (2HDMS models). We impose theoretical constraints deriving from perturbativity, stability, unitarity and correct electroweak symmetry breaking and require that the lightest CP-even Higgs, $h$, fit the LHC data for the $\sim 125.5 \mathrm{GeV}$ state at the $68 \%$ C.L. after including existing constraints from LEP and $B$ physics and LHC limits on the heavier Higgs bosons. We find that these models are easily consistent with the LUX and SuperCDMS limits on dark-matter-Nucleon scattering and the observed $\Omega h^{2}$ for $S$ masses above about $55 \mathrm{GeV}$. At lower $m_{S}$, the situation is more delicate. For points with $m_{S}$ in the $6-25 \mathrm{GeV}$ range corresponding to the CDMS II and CRESST-II positive signal ranges, the dark-matter-Nucleon cross sections predicted by the Type I and Type II models more or less automatically fall within the $95 \%-99 \%$ C.L. signal region boundaries. Were it not for the LUX and SuperCDMS limits, which exclude all (almost all) such points in the case of Type I (Type II), this would be a success for the $2 \mathrm{HDMS}$ models. In fact, in the case of Type II there are a few points with $5.5 \mathrm{GeV}$ $\lesssim m_{S} \lesssim 6.2 \mathrm{GeV}$ that survive the LUX and SuperCDMS limits and fall within the CDMS II 99\% C.L. signal region. Possibilities for dark matter to be isospin-violating in this 2HDMS context are also examined.

Keywords: Cosmology of Theories beyond the SM, Higgs Physics, Beyond Standard Model

ARXIV EPRINT: 1408.2106 


\section{Contents}

1 Introduction 1

2 Fitting the $8 \mathrm{TeV}$ LHC Higgs signal in the 2HDM 3

3 2HDMS models $\quad 5$

$\begin{array}{lll}3.1 & \text { Perturbativity } & 7\end{array}$

$\begin{array}{lll}3.2 & \text { Vacuum stability } & 7\end{array}$

$3.3 \mathcal{S}$-matrix unitarity $\quad 8$

3.4 Electroweak Symmetry Breaking (EWSB) 8

4 Experimental constraints on 2HDMS 11

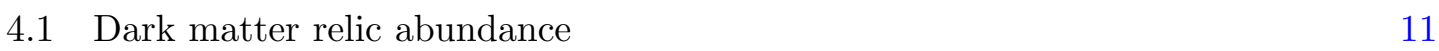

$\begin{array}{lll}4.2 & \text { Higgs invisible/unseen decays } & 14\end{array}$

$\begin{array}{lll}4.3 & \text { Direct detection } & 15\end{array}$

5 DM full mass scan $\quad 19$

$\begin{array}{lll}5.1 & \text { Type I analysis } & 20\end{array}$

5.2 Type II analysis 21

5.3 Summaries 24

5.3.1 Low mass region 26

$\begin{array}{lll}5.3 .2 & \text { Resonance region } & 27\end{array}$

$\begin{array}{lll}\text { 5.3.3 High mass region } & 27\end{array}$

6 Conclusions 28

$\begin{array}{ll}\text { A Vacuum stability } & 31\end{array}$

B Tree-level perturbative unitarity 33

\section{Introduction}

One of the most important extensions of the Standard Model (SM) is the inclusion of additional particle(s) that comprise the dark matter (DM) of the Universe. A particularly important possibility is a weakly-interacting-massive-particle (WIMP) with thermal relic density consistent with current observations. An important constraint on the WIMP scenario are limits on the spin-independent WIMP-nucleon cross section, $\sigma^{\mathrm{SI}}$, the strongest of which are currently those of the LUX [1] and SuperCDMS [2] Collaborations, where the LUX limit is strongest for DM masses above about $6 \mathrm{GeV}$ while the SuperCDMS limit is 
strongest for masses below this. ${ }^{1}$ In combination, the LUX and SuperCDMS limits exclude the positive CDMS II signal observed for a WIMP with mass of $\sim 8.6 \mathrm{GeV}$ and cross-section of $\sigma^{\mathrm{SI}} \sim 1.9 \times 10^{-41} \mathrm{~cm}^{2}[4,5]$ as well as the other positive hints (DAMA [6], CoGeNT [7, 8], and CRESST-II [9]) that support the findings of CDMS II. We note that isospin-violating DM (IVDM) scenarios [10] that could make the Xenon-based LUX limit consistent with the CDMS II Silicon-based positive signal [11-14] do not appear to be relevant given that the SuperCDMS Germanium-based limits require only minor rescaling [15, 16].

In this paper we focus on a one-component DM model in which the WIMP is a singlet scalar particle that is present as part of an extended scalar sector of the electroweak theory. In particular, we consider two-Higgs-doublet models (2HDM) with an extra real scalar $S$ (we term the resulting models "2HDMS") that is neutral under the SM gauge group. ${ }^{2}$ We introduce an extra $\mathbb{Z}_{2}^{\prime}$ symmetry under which $S$ is the only odd field. Provided $S$ does not acquire a vacuum expectation value $(\mathrm{VEV})$, it is stable and thereby a possible DM candidate. The 2HDMS then contains three CP-even states, $h$ and $H\left(m_{h} \leq m_{H}\right)$ from the $2 \mathrm{HDM}$ sector and $S$, a CP-odd state, $A$, and a charged Higgs pair, $H^{ \pm}$. The $2 \mathrm{HDM}$ context allows for increased flexibility for DM predictions as compared to adding an $S$ to the one-doublet SM in that either $h$ or $H$ can be identified with the observed SM-like CPeven state at $\sim 125.5 \mathrm{GeV}$ while the other $\mathrm{CP}$-even state and the $A$ and $H^{ \pm}$can provide additional channels for early-universe annihilation. Further, both of the CP-even states contribute to DM scattering and annihilation.

That the 2HDM can provide a consistent description of all LHC observed signal strengths for either the $h$ or $H$ identified as the observed $\sim 125.5 \mathrm{GeV}$ state (for the Type I or Type II version of the model) is well-known [17-34]. For simplicity, in this paper we consider only the case of $m_{h} \sim 125.5 \mathrm{GeV}$. In the context of DM, the crucial new ingredient offered by 2HDMS is the presence of two independent Higgs portal couplings, $H_{1}^{\dagger} H_{1} S S$ and $H_{2}^{\dagger} H_{2} S S$, where $H_{1,2}$ are the two Higgs doublets of the 2HDM. As will be discussed in detail later, this is an important feature that makes it possible to decouple DM annihilation from DM scattering off nucleons. It also provides more freedom while trying to overcome constraints from invisible decays of the $125.5 \mathrm{GeV}$ Higgs boson in a multiple scalar singlet extension of the SM [35] or in the two component DM scenario of [36]. The singlet extension of the 2HDM has been studied earlier in [37-45] and also discussed in the frameworks of scale invariance [46] and non-SUSY SO(10) grand unification (GUT) [4749]. The primary new ingredient in the present paper is the inclusion of the full set of constraints on the $2 \mathrm{HDM}$ sector of the $2 \mathrm{HDMS}$. These include: requiring consistency with "preLHC" constraints; an accurate fit to the combined ATLAS and CMS Higgs signal data when the $h$ is identified with the $\sim 125.5 \mathrm{GeV}$ state; and enforcing LHC limits on the other Higgs bosons ( $H, A$ and $H^{ \pm}$) of the $2 \mathrm{HDM}$ using the procedures of [32]. With regard to the singlet sector, we derive and employ the constraints on the singlet parameters resulting

\footnotetext{
${ }^{1}$ We note that the XENON 100 limit [3] is weaker than the LUX limit for all dark-matter masses and, thus, we do not reference it in our discussions.

${ }^{2}$ Here we will restrict ourself to the CP-conserving version of the 2 HDM. However, this assumption is just to reduce the number of parameters. The analysis could as well be performed assuming either spontaneous or explicit violation of $\mathrm{CP}$ in the scalar sector.
} 
from imposing perturbativity, stability, unitarity and correct electroweak symmetry breaking. As implicit from the $H_{1}^{\dagger} H_{1} S S$ and $H_{2}^{\dagger} H_{2} S S$ Higgs portal interactions, $S S$ annihilation via both the $h$ pole and the $H$ pole will be accounted for.

In the following section, we will summarize the fits to preLHC and LHC data within the $2 \mathrm{HDM}$ context with $m_{h} \sim 125.5 \mathrm{GeV}$. In section 3 , we discuss the two-Higgs-doublets plus singlet model (2HDMS), including its general features and theoretical constraints as well as the properties of the singlet dark matter scalar. In section 4 we elaborate on the methodology of constraining the full 2HDMS parameter space using various experimental observations and limits when the $2 \mathrm{HDM}$ sector of the model is restricted to fit existing LHC data. In section 5 we will present the results of our 2HDMS parameter space scan. There, we show that the combined LUX and SuperCDMS DM limits can only be satisfied for $m_{S} \gtrsim 55 \mathrm{GeV}$. However, we do explore the extent to which IVDM scenarios arise in the 2HDMS case and how they come close to allowing the CDMS II signal to be consistent with the LUX limit. Section 6 contains our conclusions. In appendices A and B we derive the constraints on the 2HDMS from vacuum stability and unitarity, respectively.

\section{Fitting the $8 \mathrm{TeV}$ LHC Higgs signal in the 2HDM}

The combined ATLAS and CMS data imply that the observed $\sim 125.5 \mathrm{GeV}$ state is quite consistent with SM-like Higgs boson. Recent 2HDM efforts [17-34] have thus focused on the extent to which deviations from the SM are still possible and the implications for possibly observing such deviations and/or the other Higgs bosons in future LHC running. Of course, one must keep in mind that there is still an enhanced $\gamma \gamma$ signal in the ATLAS analysis whereas $\gamma \gamma$ rates are somewhat suppressed according to the CMS analysis and it is only the combined results that show no $\gamma \gamma$ enhancement. Should an enhancement become statistically certain in future LHC runs, this could certainly be accommodated in the $2 \mathrm{HDM}$ context [50-59], as could a suppression, but the analysis performed in this paper would have to be revisited. In this paper, we take the combined data at face value and employ the very recent $2 \mathrm{HDM}$ fits of [32] keeping only points that are consistent with observations at the (rather stringent) 68\% C.L., assuming that it is the lighter $h$ that should be identified with the observed $\sim 125.5 \mathrm{GeV}$ state. To be specific, the predicted signal strengths in the $\mu(\mathrm{ggF}+\mathrm{ttH})$ versus $\mu(\mathrm{VBF}+\mathrm{VH})$ planes for each of the $\gamma \gamma, V V$ (where $V V \equiv Z Z, W W), b \bar{b}$, and $\tau \tau$ final states were required to have $\chi^{2}<2.3$ as determined using the C.L. contours established in [60].

The parameters of $2 \mathrm{HDM}$ can be taken to be the mixing angle, $\alpha$, that diagonalizes the CP-even scalar sector, ${ }^{3} \tan \beta=v_{2} / v_{1}$ where $v_{1,2}=\left\langle H_{1,2}\right\rangle\left(\right.$ with $\left.v_{1}^{2}+v_{2}^{2}=(246 \mathrm{GeV})^{2}\right)$, the masses of the physical Higgs bosons, $m_{h}, m_{H}, m_{H^{ \pm}}$, and $m_{12}^{2}$, where $m_{12}^{2}$ specifies the soft breaking of the $\mathbb{Z}_{2}$ symmetry needed to protect the $2 \mathrm{HDM}$ from tree-level flavor-changing neutral currents (FCNC). The most popular 2HDM's that are free of FCNC are the Type I and Type II models. In Type I, quark masses and Yukawa couplings derive only from one Higgs doublet, conventionally chosen to be $H_{2}$. In Type II, up quarks couple only to $H_{2}$ and down quarks and leptons couple only to $H_{1}$. The couplings, normalized to their SM

\footnotetext{
${ }^{3}$ We follow the conventions of [61].
} 


\begin{tabular}{|c|c|c|c|c|c|}
\hline & Type I and II & \multicolumn{2}{|c|}{ Type I } & \multicolumn{2}{c|}{ Type II } \\
\hline Higgs & $C_{V}$ & $C_{U}$ & $C_{D}$ & $C_{U}$ & $C_{D}$ \\
\hline$h$ & $\sin (\beta-\alpha)$ & $\cos \alpha / \sin \beta$ & $\cos \alpha / \sin \beta$ & $\cos \alpha / \sin \beta$ & $-\sin \alpha / \cos \beta$ \\
\hline$H$ & $\cos (\beta-\alpha)$ & $\sin \alpha / \sin \beta$ & $\sin \alpha / \sin \beta$ & $\sin \alpha / \sin \beta$ & $\cos \alpha / \cos \beta$ \\
\hline$A$ & 0 & $\cot \beta$ & $-\cot \beta$ & $\cot \beta$ & $\tan \beta$ \\
\hline
\end{tabular}

Table 1. Tree-level vector boson couplings $C_{V}(V=W, Z)$ and fermionic couplings $C_{F}(F=U, D)$ normalized to their SM values for the Type I and Type II two-Higgs-doublet models.
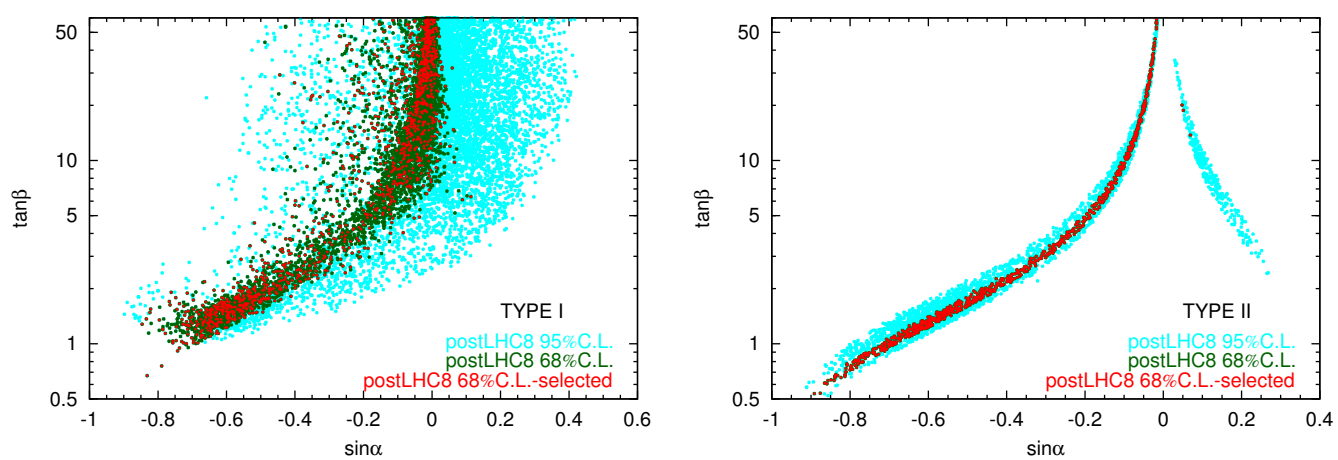

Figure 1. $2 \mathrm{HDM}$ points in the $(\tan \beta, \sin \alpha)$ plane that provide a fit the LHC/Tevatron signal strengths at $95 \%$ C.L. (cyan) and $68 \%$ C.L. (dark green), from the analysis in [60]. In red we have marked the $68 \%$ C.L. points used later in the singlet scalar model analysis (for the Type II model we have used all $68 \%$ C.L. points).

values, of the Higgs bosons to vector bosons $\left(C_{V}\right)$ and to up- and down-type fermions $\left(C_{U}\right.$ and $C_{D}$ ) are functions of $\alpha$ and $\beta$ as given in table 1; see e.g. [62] for details. The Type I and Type II models are distinguished only by the pattern of their fermionic couplings.

When expanding the $2 \mathrm{HDM}$ to include an extra singlet that could be dark matter, it is appropriate to begin with $2 \mathrm{HDM}$ points that provide a good fit to the LHC data. As noted above, we assume that it is the lighter $h$ that should be identified with the $125.5 \mathrm{GeV}$ state and take the $2 \mathrm{HDM}$ points from [32] that provide a fit to the LHC data within $68 \%$ C.L. These points, along with the points agreeing at the less restrictive 95\% C.L., are shown in figure 1 using the $\tan \beta$ vs. $\sin \alpha$ plane. (Because there are so many $68 \%$ C.L. points in the Type I $2 \mathrm{HDM}$ we employ only a subset of these points in this case - the full $68 \%$ C.L. set of points are shown in dark green while the selected points are shown in red.) Of course, in order that the LHC fit for $m_{h} \sim 125.5 \mathrm{GeV}$ be good, the vector boson and fermionic couplings (see table 1) should be quite SM-like. The exact SM limit occurs for $\beta-\alpha=\pi / 2$. The extent to which $68 \%$ C.L. allows deviation in these couplings is illustrated in figure 2 where we plot the ratios of these couplings to their SM values, $C_{V}^{h}$ for the $V V$ coupling and $C_{D}^{h}$ for the down-quark. (For Type I, $C_{U}^{h}=C_{D}^{h}$.) We observe that in the case of Type II almost all points have $C_{V}^{h}$ and $C_{D}^{h}$ (and $C_{U}^{h}$, not plotted) very close to unity (whereas at 95\% C.L. significant deviations are allowed). In the case of Type I, significant deviations in these couplings from unity are still allowed at $68 \%$ C.L.

When adding in the singlet $S$ we thus must be certain that it will not significantly disturb the fit of the $h$ to the LHC data. Because of the extra imposed $\mathbb{Z}^{\prime}{ }_{2}$ symmetry, 

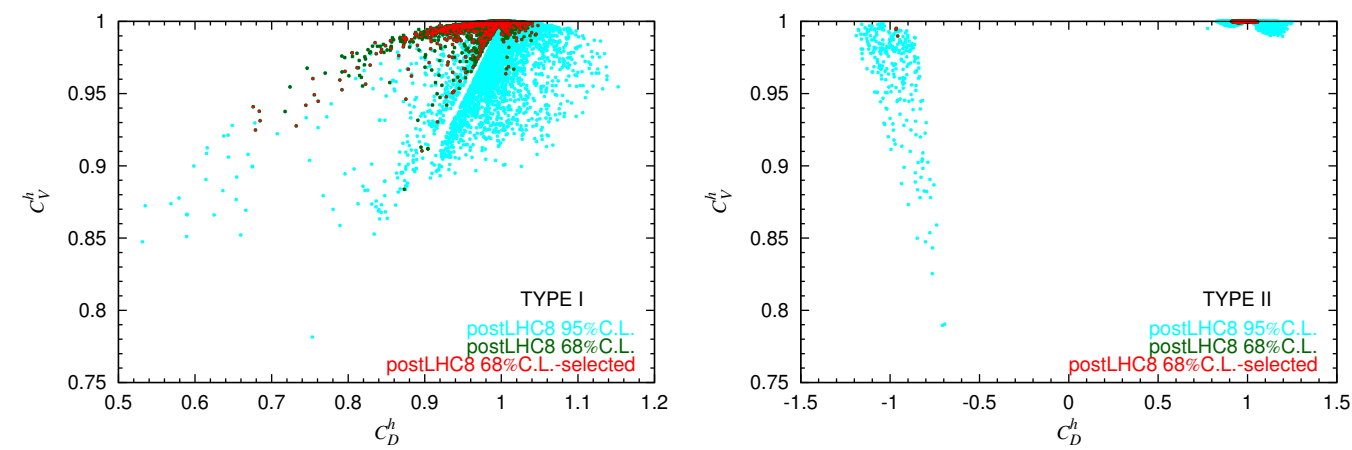

Figure 2. $2 \mathrm{HDM}$ points in the $\left(C_{V}^{h}, C_{D}^{h}\right)$ plane using the notation of figure 1. For Type I, $C_{U}^{h}=C_{D}^{h}$.

the only influence of the $S$ on the $h$ fits arises if $h \rightarrow S S$ decays are present, which of course requires $m_{S}<m_{h} / 2$. These would constitute invisible decays. In [60] a 68\% C.L. limit of $\mathrm{BR}_{\text {inv }} \leq 0.1$ (see also $[63,64]$ ) was obtained in the context where the $C_{U}, C_{D}$ and $C_{V}$ coupling ratios could be varied with respect to their SM values of unity (but with $C_{V} \leq 1$ as appropriate to a $2 \mathrm{HDM}$ ) and assuming no extra loop contributions to the $h \gamma \gamma$ and $h g g$ couplings. In the $2 \mathrm{HDM}$, the $H^{ \pm}$loops can contribute to the $h \gamma \gamma$ coupling, but for simplicity we will assume that $\mathrm{BR}_{\text {inv }} \leq 0.1$ remains applicable. The constraint of small $\mathrm{BR}_{\text {inv }}=\mathrm{BR}(h \rightarrow S S)$ plays a major role in eliminating many $m_{S}<m_{h} / 2$ scenarios.

\section{2HDMS models}

Our goal is to analyse a model with two Higgs doublets $H_{1}, H_{2}$ and a real scalar $S$, which is a singlet under the SM gauge group. We will assign equal $\mathrm{U}(1)_{Y}$ charges $Y=1$ to $H_{1}$ and $H_{2}$. We also introduce a $\mathbb{Z}_{2}^{\prime}$ symmetry under which $S \rightarrow-S$ (other fields are taken to be even under $\mathbb{Z}^{\prime}$ ). We call this model 2 HDMS. ${ }^{4}$ The most general gauge-invariant $2 \mathrm{HDMS}$ scalar potential is then:

$$
\begin{aligned}
V\left(H_{1}, H_{2}, S\right)= & m_{1}^{2} H_{1}^{\dagger} H_{1}+m_{2}^{2} H_{2}^{\dagger} H_{2}-\left[m_{12}^{2} H_{1}^{\dagger} H_{2}+\text { h.c. }\right] \\
& +\frac{\lambda_{1}}{2}\left(H_{1}^{\dagger} H_{1}\right)^{2}+\frac{\lambda_{2}}{2}\left(H_{2}^{\dagger} H_{2}\right)^{2}+\lambda_{3}\left(H_{1}^{\dagger} H_{1}\right)\left(H_{2}^{\dagger} H_{2}\right)+\lambda_{4}\left|H_{1}^{\dagger} H_{2}\right|^{2} \\
& +\left[\frac{\lambda_{5}}{2}\left(H_{1}^{\dagger} H_{2}\right)^{2}+\lambda_{6}\left(H_{1}^{\dagger} H_{1}\right)\left(H_{1}^{\dagger} H_{2}\right)+\lambda_{7}\left(H_{2}^{\dagger} H_{2}\right)\left(H_{1}^{\dagger} H_{2}\right)+\text { h.c. }\right] \\
& +\frac{1}{2} m_{0}^{2} S^{2}+\frac{1}{4 !} \lambda_{S} S^{4}+\kappa_{1} S^{2}\left(H_{1}^{\dagger} H_{1}\right)+\kappa_{2} S^{2}\left(H_{2}^{\dagger} H_{2}\right)+S^{2}\left(\kappa_{3} H_{1}^{\dagger} H_{2}+\text { h.c. }\right)
\end{aligned}
$$

which contains 20 (real) parameters. However, for simplicity we make several additional assumptions. We consider a model without explicit $\mathrm{CP}$ violation (i.e. all the $\lambda$ coefficients of eq. (3.1) are taken to be real) and we only consider parameter choices for which there is no spontaneous CP breaking. As a result, the Higgs VEVs are real. We also impose a $\mathbb{Z}_{2}$ symmetry under which $H_{1} \rightarrow H_{1}, H_{2} \rightarrow-H_{2}, S \rightarrow S$. This eliminates the $\lambda_{6}, \lambda_{7}$ and $\kappa_{3}$

\footnotetext{
${ }^{4}$ This model was referred to as the 2HDM Darkon model (2HDMD) in some earlier literature.
} 
couplings of eq. (3.1). However, we do allow for $m_{12}^{2} \neq 0$, corresponding to a soft breaking of $\mathbb{Z}_{2}$. The resulting potential takes the form

$$
\begin{aligned}
V\left(H_{1}, H_{2}, S\right)= & m_{1}^{2} H_{1}^{\dagger} H_{1}+m_{2}^{2} H_{2}^{\dagger} H_{2}-\left[m_{12}^{2} H_{1}^{\dagger} H_{2}+\text { h.c. }\right]+\frac{\lambda_{1}}{2}\left(H_{1}^{\dagger} H_{1}\right)^{2}+\frac{\lambda_{2}}{2}\left(H_{2}^{\dagger} H_{2}\right)^{2} \\
& +\lambda_{3}\left(H_{1}^{\dagger} H_{1}\right)\left(H_{2}^{\dagger} H_{2}\right)+\lambda_{4}\left|H_{1}^{\dagger} H_{2}\right|^{2}+\left[\frac{\lambda_{5}}{2}\left(H_{1}^{\dagger} H_{2}\right)^{2}+\text { h.c. }\right] \\
& +\frac{1}{2} m_{0}^{2} S^{2}+\frac{1}{4 !} \lambda_{S} S^{4}+\kappa_{1} S^{2}\left(H_{1}^{\dagger} H_{1}\right)+\kappa_{2} S^{2}\left(H_{2}^{\dagger} H_{2}\right)
\end{aligned}
$$

The next stage is to convert from the Lagrangian basis to the mass eigenstate basis. Despite the presence of the $S^{2} H_{1}^{\dagger} H_{1}$ and $S^{2} H_{2}^{\dagger} H_{2}$ terms, the analysis of the 2HDM sector can be performed independently of the $S$ and the usual mass matrices for the 2HDM, see [61], are not changed due to the fact that the extra field $S$ does not acquire a VEV. ${ }^{5}$ However, the fields $H_{1}$ and $H_{2}$ do contribute to the $S^{2}$ mass term when they develop VEVs, $H_{1,2} \rightarrow v_{1,2}$.

In terms of the mass eigenstates, the $S$-dependent part of the scalar potential has the form:

$$
\begin{aligned}
-V_{S}=-\frac{1}{2} m_{S}^{2} S^{2} & -\lambda_{h} v h S^{2}-\lambda_{H} v H S^{2} \\
& -S^{2}\left(\lambda_{H H} H H+\lambda_{h H} h H+\lambda_{h h} h h+\lambda_{A A} A A+\lambda_{H^{+} H^{-}} H^{+} H^{-}\right)
\end{aligned}
$$

where the physical $S$ particle mass and the DM-Higgs trilinear couplings are

$$
\begin{aligned}
m_{S}^{2} & =m_{0}^{2}+\left(\kappa_{1} \cos ^{2} \beta+\kappa_{2} \sin ^{2} \beta\right) v^{2} \\
\lambda_{h} & =-\kappa_{1} \sin \alpha \cos \beta+\kappa_{2} \cos \alpha \sin \beta \\
\lambda_{H} & =\kappa_{1} \cos \alpha \cos \beta+\kappa_{2} \sin \alpha \sin \beta .
\end{aligned}
$$

While $m_{0}, \kappa_{1}$ and $\kappa_{2}$ constitute a complete set of extra (as compared to the 2HDM) free parameters for the scalar sector of the 2HDMS Lagrangian, in practice it is more convenient to employ the DM mass $m_{S}$ and the couplings $\lambda_{h}$ and $\lambda_{H}$ as the new independent set of free parameters associated with the $S$ sector. In the limit of $\sin (\beta-\alpha)=1$, for which the $h$ has exactly SM-like couplings to $V V$ and $f \bar{f}$,

$$
\begin{aligned}
\lambda_{h} & =\kappa_{1} \cos ^{2} \beta+\kappa_{2} \sin ^{2} \beta, \\
\lambda_{H} & =\left(\kappa_{1}-\kappa_{2}\right) \sin \beta \cos \beta .
\end{aligned}
$$

We also emphasize that although there is no $A S S$ term in $V_{S}$ due to $\mathrm{CP}$, the CP-odd Higgs boson $A$ still plays a role in determining the DM relic density through the creation/annihilation process $S S \longleftrightarrow A A$. We will discuss this issue in section 4 .

\footnotetext{
${ }^{5}$ If $S$ acquires a VEV spontaneously, as considered in [45], the $S$ mixes with the doublet Higgs and cannot be dark matter.
} 
The quadrilinear couplings $\lambda_{H H}, \lambda_{h H}, \lambda_{h h}, \lambda_{A A}, \lambda_{H^{+} H^{-}}$can also be expressed in terms of the $\kappa_{1}, \kappa_{2}, \alpha$ and $\beta$ parameters:

$$
\begin{aligned}
\lambda_{A A} & =\frac{1}{2} \lambda_{H^{+} H^{-}}=\frac{1}{2}\left(\kappa_{1} \sin ^{2} \beta+\kappa_{2} \cos ^{2} \beta\right) \\
\lambda_{h h} & =\frac{1}{2}\left(\kappa_{2} \cos ^{2} \alpha+\kappa_{1} \sin ^{2} \alpha\right), \quad \lambda_{H H}=\frac{1}{2}\left(\kappa_{1} \cos ^{2} \alpha+\kappa_{2} \sin ^{2} \alpha\right), \\
\lambda_{h H} & =\frac{1}{2}\left(\kappa_{2}-\kappa_{1}\right) \sin 2 \alpha .
\end{aligned}
$$

We note that the above Lagrangian-level trilinear and quadrilinear couplings convert to Feynman rules according to:

$$
\begin{aligned}
g_{h S S, H S S} & =-2 \lambda_{h, H} v, & g_{h h S, H H S S} & =-4 \lambda_{h h, H H}, \\
g_{h H S S} & =-2 \lambda_{h H}, & g_{A A S S} & =g_{H^{+} H^{-} S S}=-4 \lambda_{A A} .
\end{aligned}
$$

The fermionic couplings in the 2HDMS depend upon the behavior of the fermionic fields under $\mathbb{Z}_{2}$ and $\mathbb{Z}_{2}^{\prime}$. We assume that the fermions are even under $\mathbb{Z}_{2}^{\prime}$ so that the $S$ has no tri-linear coupling to $f \bar{f}$. ${ }^{6}$ Fermionic couplings to $H_{1}$ and $H_{2}$ depend on the $\mathbb{Z}_{2}$ signs for fermions. We choose these so as to forbid flavour-changing Yukawa couplings for the neutral Higgs bosons, resulting in the couplings of table 1 for the models of Type I and Type II. From now on, we restrict ourselves to the $\mathbb{Z}_{2} \times \mathbb{Z}^{\prime}{ }_{2}$ case.

Further constraints on the model are as follows.

\subsection{Perturbativity}

All quartic Feynman rules associated with the mass eigenstates $h, H, A, H^{ \pm}, S$ are required to satisfy the standard perturbativity constraint, i.e. their absolute values must be $\leq 4 \pi$. As regards the sector involving the $S$ field, the quartic couplings of interest are those in which $S^{2}$ multiplies two 2HDM fields and the $S^{4}$ term. One can show that the quartic Feynman rules connecting $S^{2}$ to two neutral $2 \mathrm{HDM}$ fields, summarized above, are guaranteed to be smaller than $4 \pi$ in absolute value if $\left|\kappa_{1}\right|,\left|\kappa_{2}\right| \leq 4 \pi$ is imposed. However, these maximum values are only allowed for $\alpha= \pm \pi / 4$. The Feynman rule for $S^{4}$ interactions being $\lambda_{S}$ means that we must also impose $0<\lambda_{S} \leq 4 \pi$, the lower bound being that required for stability.

\subsection{Vacuum stability}

We require that the vacuum is stable at tree level, which means that the potential in (3.2) has to be bounded from below. As already noted this requires first of all that $\lambda_{S}>0$. Given this, it is shown in appendix A that the necessary and sufficient conditions for stability read:

$$
\begin{aligned}
& \lambda_{1}, \lambda_{2}, \lambda_{S}>0, \\
& \lambda_{3}+\lambda_{4}-\left|\lambda_{5}\right|>-\sqrt{\lambda_{1} \lambda_{2}}, \quad \lambda_{3}>-\sqrt{\lambda_{1} \lambda_{2}} \\
& \kappa_{1}>-\sqrt{\frac{1}{12} \lambda_{S} \lambda_{1}}, \\
& \kappa_{2}>-\sqrt{\frac{1}{12} \lambda_{S} \lambda_{2}} .
\end{aligned}
$$

\footnotetext{
${ }^{6}$ We do not consider here the possibility of coupling the singlet to the Majorana mass term for righthanded neutrinos, $s \nu_{R i}^{T} C \nu_{R j}$ for $i \neq j$. In fact such couplings are allowed if $\nu_{R i}$ carry $\mathbb{Z}_{2}^{\prime}$ charge, see [65].
} 
If $\kappa_{1}$ or $\kappa_{2}<0$, then we have to satisfy also:

$$
\begin{aligned}
-2 \kappa_{1} \kappa_{2}+\frac{1}{6} \lambda_{S} \lambda_{3} & >-\sqrt{4\left(\frac{1}{12} \lambda_{S} \lambda_{1}-\kappa_{1}^{2}\right)\left(\frac{1}{12} \lambda_{S} \lambda_{2}-\kappa_{2}^{2}\right)} \\
-2 \kappa_{1} \kappa_{2}+\frac{1}{6} \lambda_{S}\left(\lambda_{3}+\lambda_{4}-\left|\lambda_{5}\right|\right) & >-\sqrt{4\left(\frac{1}{12} \lambda_{S} \lambda_{1}-\kappa_{1}^{2}\right)\left(\frac{1}{12} \lambda_{S} \lambda_{2}-\kappa_{2}^{2}\right)} .
\end{aligned}
$$

The conditions in eq. (3.12) above are the standard 2HDM stability conditions. These are supplemented by the requirements of eq. (3.13), eq. (3.14) and eq. (3.15) in the presence of the singlet field.

\section{$3.3 \mathcal{S}$-matrix unitarity}

In addition, there are constraints deriving from unitarity that are closely correlated with the constraints from perturbativity. Indeed, the dominant non-vanishing contributions to amplitudes for two-body scattering at high energy come from the processes mediated by quartic couplings. Therefore, the unitarity constraint for $J=0$ partial waves, $\left|a_{0}\right| \leq 1 / 2$, reduces to a constraint on these quartic couplings. In appendix $\mathrm{B}$, we describe in more detail the unitarity bounds and give explicit formulae for the scattering matrix of two-body processes in the scalar sector of the 2 HDMS model. ${ }^{7}$

\subsection{Electroweak Symmetry Breaking (EWSB)}

In order to ensure a stable DM particle $S$, one has to require $\langle S\rangle=0$ at the global minimum of the scalar potential, eq. (3.2). For each 2 HDM point at $68 \%$ C.L. (marked in red in figure 1), $\tan \beta$ and $m_{12}^{2}$ are given and all five $\lambda$ 's can be computed from the masses of the Higgs bosons and $\sin \alpha$ (see details in appendix D of [61]). With these specified, the remaining parameters $m_{1}$ and $m_{2}$ in the potential, eq. (3.2), are determined by the minimization conditions

$$
\begin{aligned}
& m_{1}^{2}=m_{12}^{2} \tan \beta-\frac{1}{2} v^{2}\left(\lambda_{1} \cos ^{2} \beta+\left(\lambda_{3}+\lambda_{4}+\lambda_{5}\right) \sin ^{2} \beta\right) \\
& m_{2}^{2}=m_{12}^{2} \cot \beta-\frac{1}{2} v^{2}\left(\lambda_{2} \sin ^{2} \beta+\left(\lambda_{3}+\lambda_{4}+\lambda_{5}\right) \cos ^{2} \beta\right) .
\end{aligned}
$$

Note that the minimization with respect to $S$ is trivial because of $\langle S\rangle=0$. In practice, we find all the minima of eq. (3.2) numerically and then eliminate the points for which the global minimum is not at $\langle S\rangle=0,\left\langle H_{1}\right\rangle \neq 0,\left\langle H_{2}\right\rangle \neq 0$.

In figure 3 , the allowed regions in the $\left(\kappa_{1}, \kappa_{2}\right)$ parameter space are displayed after sequentially imposing the various constraints discussed above.

i) At the first level, we impose perturbativity (P). All subsequent point layers obey P.

\footnotetext{
${ }^{7}$ It is important to note that the $2 \rightarrow 2$ scattering matrix that is obtained when $S$-related channels are included always has a maximum eigenvalue that is larger than that of the pure 2HDM $2 \rightarrow 2$ scattering matrix. This is called the "bordering theorem" (see e.g. [66]). Thus, although our 2HDM points have already been filtered using the 2HDMC code [67, 68] which imposes unitarity in the 2HDM context, the unitarity limits obtained after including the $S$-related channels are guaranteed to be stronger.
} 

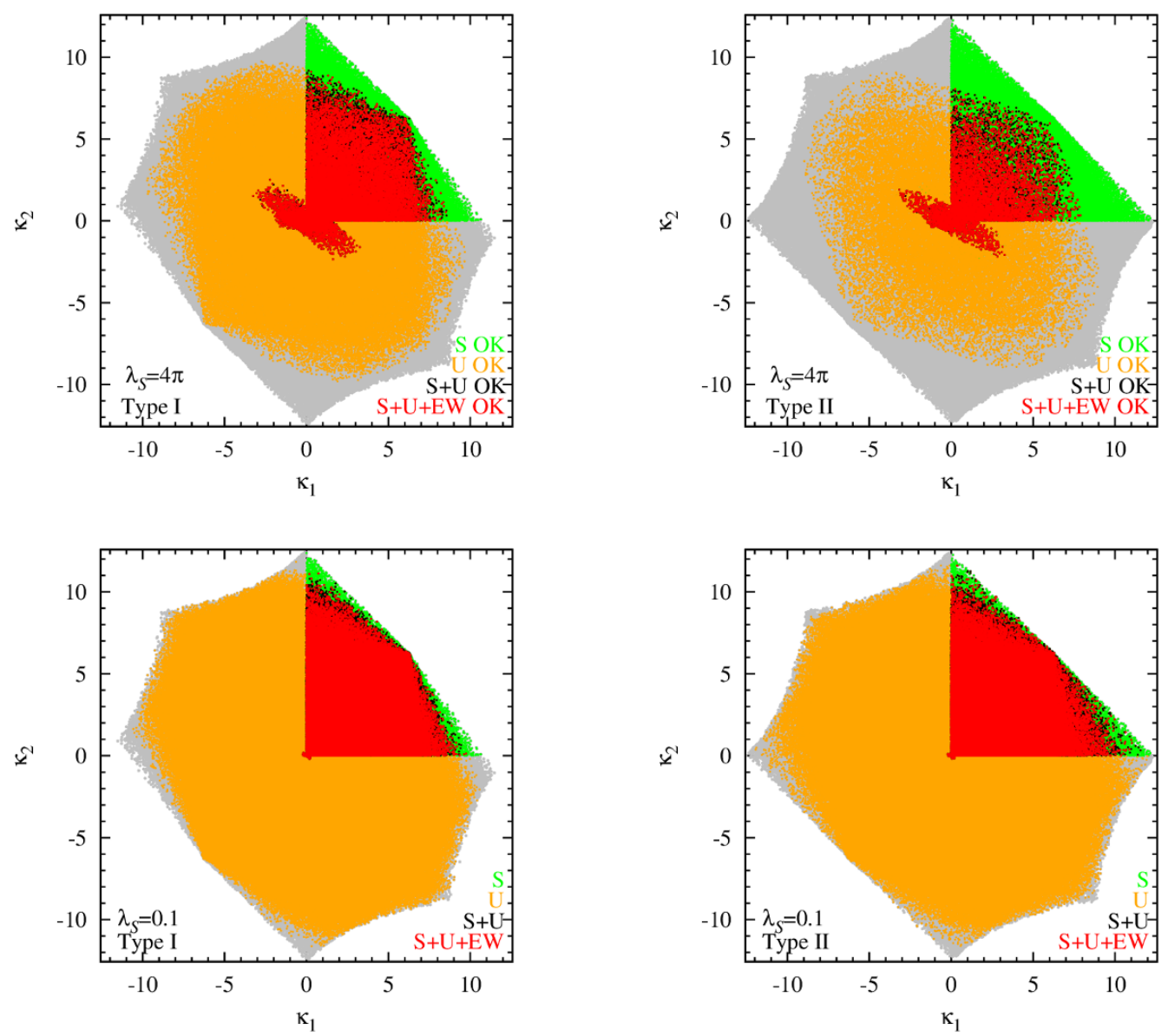

Figure 3. The plot shows the impact of the perturbativity (P), vacuum stability (S), unitarity (U) and electroweak symmetry breaking (EW) global minimum bounds discussed in section 3 on the $\left(\kappa_{1}, \kappa_{2}\right)$ plane. At the first level, the grey points are those which satisfy $\mathrm{P}$ - all subsequent point layers obey P. Note that $\left|\kappa_{1}\right|,\left|\kappa_{2}\right| \leq 4 \pi$ contains the perturbative region (see section 3.1). Subsequent point layers were plotted in the following order: points after the stability bound, $\mathrm{S}$ (green), points after the unitarity bound, U (orange), points after the stability and unitarity bounds, $\mathrm{S}+\mathrm{U}$ (black), points after the stability, unitarity and EW bounds, $\mathrm{S}+\mathrm{U}+\mathrm{EW}$ (red). The value of the $\lambda_{S}$ parameter was set to $4 \pi(0.1)$ in the upper (lower) plots. In this figure, no restriction on $m_{S}$ is imposed.

ii) Next, we require vacuum stability (S).

$\mathrm{S}$ is always guaranteed as long as $\kappa_{1}$ and $\kappa_{2}$ are both positive. For $\kappa_{1}<0$ and/or $\kappa_{2}<0$, vacuum stability depends on the value of the $S$ self-interaction coupling $\lambda_{S}$.

Choosing the maximum value of $\lambda_{S}=4 \pi$ (upper panel), there is an ellipse-shaped region of modest size where $\kappa_{1}$ and/or $\kappa_{2}$ can be negative. This ellipse-shaped region shrinks as $\lambda_{S}$ decreases — we illustrate this for the case of $\lambda_{S}=0.1$ in the lower panel.

iii) Third, the unitarity conditions (U) on their own produce an oval-shaped region in the $\kappa_{2}$ vs. $\kappa_{1}$ plane. 

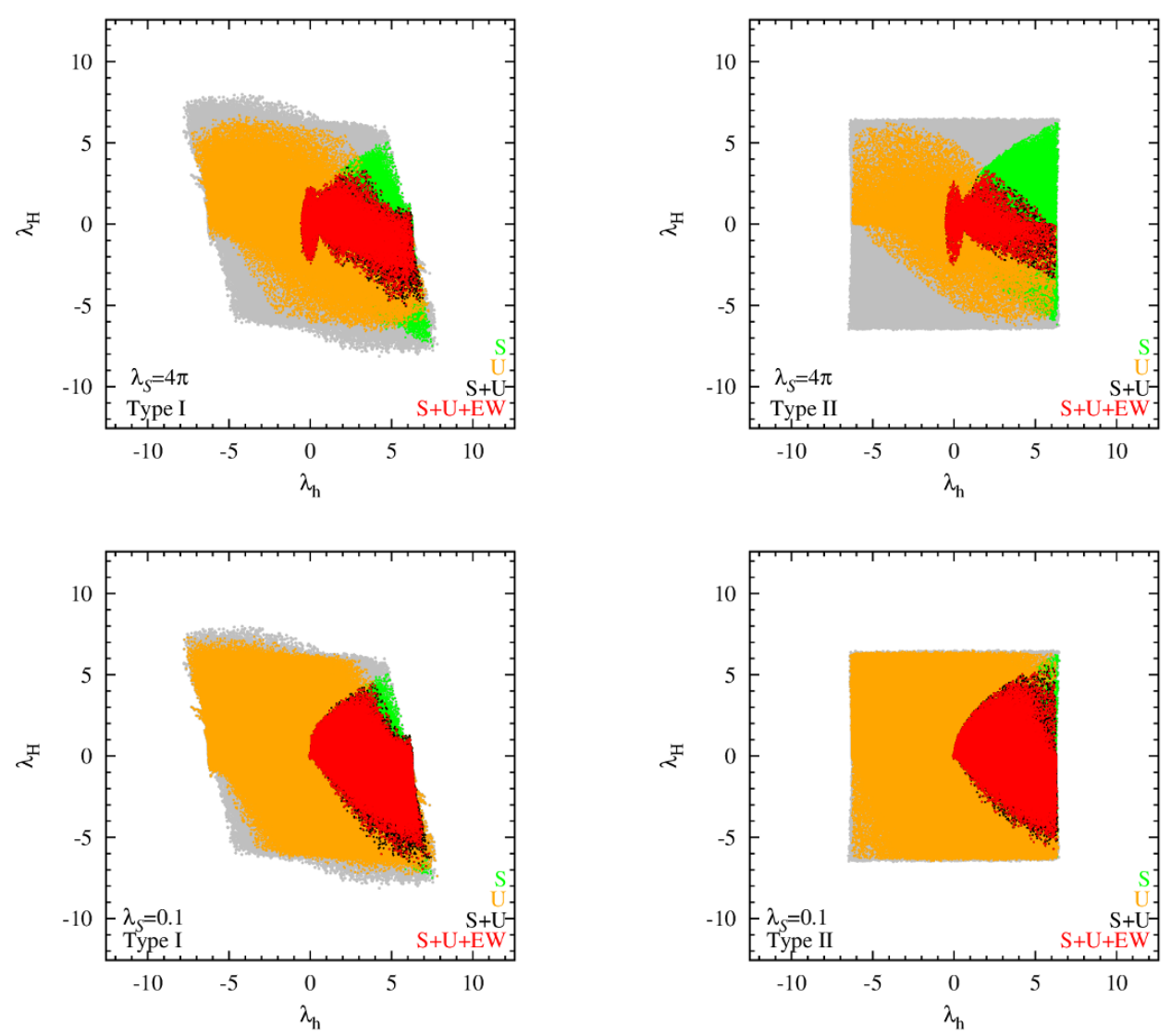

Figure 4. Bounds in the $\left(\lambda_{h}, \lambda_{H}\right)$ plane associated with the sequential constraints as described in the caption for figure 3 . No restriction on $m_{S}$ is imposed.

iv) If both $\mathrm{S}$ and $\mathrm{U}$ are imposed we are left with the black points (all red points are also black points).

v) Imposing $\mathrm{S}+\mathrm{U}+\mathrm{EW}$ eliminates some of the (black) $\mathrm{S}+\mathrm{U}$ points, leaving us with the red points.

Figure 4 shows how the above $\kappa_{1}$ vs. $\kappa_{2}$ regions map into the $\lambda_{h}$ vs. $\lambda_{H}$ parameter space. In this figure, no restriction on $m_{S}$ is imposed. In fact, the $\mathrm{P}+\mathrm{S}+\mathrm{U}+\mathrm{EW}$ constraints are much more restrictive for $m_{S}<m_{h} / 2$. This is illustrated in figure 5. In particular, note that the maximum value of $\lambda_{H}$ that is allowed is of order 3 in magnitude, at large $\lambda_{S}$, and is very tiny for small $\lambda_{S}$. As a result, very large values of $m_{H}$ cannot result in sufficient annihilation through the $H$ pole diagram when $m_{S}<m_{h} / 2$ given that the $h$ pole diagram is suppressed because $\lambda_{h}$ must be very small in order to avoid too large $\mathrm{BR}(h \rightarrow S S)$.

Of course, $\mathrm{P}+\mathrm{S}+\mathrm{U}+\mathrm{EW}$ are only the most basic constraints. In the following sections, we will show that once $\Omega h^{2}$ is required to agree with observations, then $\left|\lambda_{h}\right|$ and $\left|\lambda_{H}\right|$ are restricted to values $\lesssim 0.2$ and $\lesssim 2.5$, respectively. When $m_{S}<m_{h} / 2, \operatorname{BR}(h \rightarrow S S) \leq 0.1$ further constrains $\left|\lambda_{h}\right|$ to values $\lesssim 0.01$. 

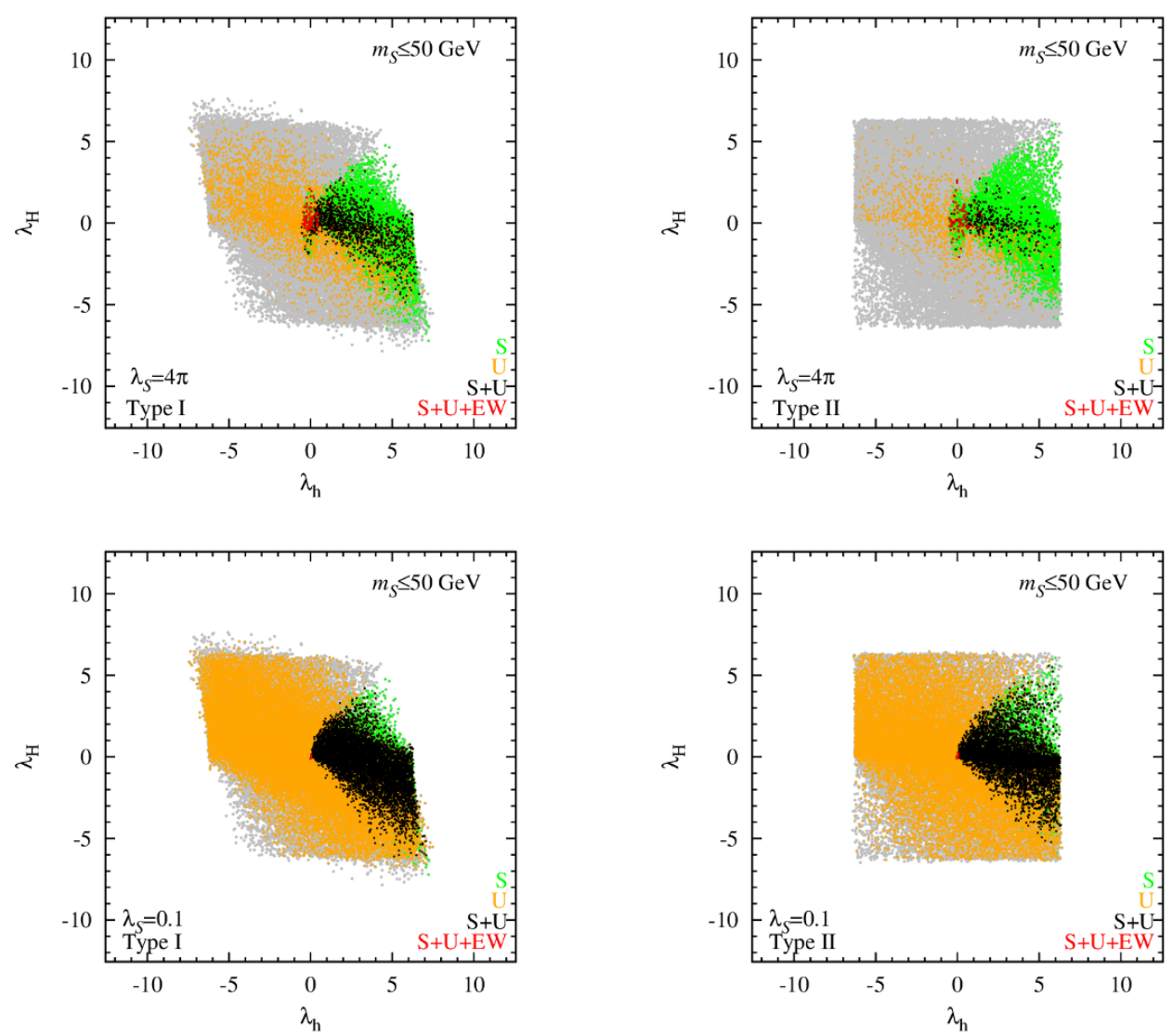

Figure 5. Bounds in the $\left(\lambda_{h}, \lambda_{H}\right)$ plane associated with the sequential constraints as described in the caption for figure 3 for parameter choices yielding $m_{S} \leq 50 \mathrm{GeV}$. We observe that EW is an especially strong contraint in this mass region.

\section{Experimental constraints on 2HDMS}

Before starting our analysis of the model, we would like to summarize the experiments that impact the extra singlet $S$ particle.

\subsection{Dark matter relic abundance}

In the 2HDMS, the $S$ particle provides the only candidate for DM and thus should comprise the total relic abundance of the early Universe. To a good approximation, the relic density is given by

$$
\Omega_{S} \simeq 1.07 \times 10^{9} \frac{x_{f}}{\sqrt{g_{*}} M_{\mathrm{Pl}}\left\langle\sigma_{\mathrm{ann}} v_{\mathrm{rel}}\right\rangle} \mathrm{GeV}^{-1}
$$

where $x_{f}=m_{S} / T_{f} \simeq 20$ is the typical freeze-out temperature of a WIMP [69], $M_{P l}$ is the Planck mass, $g_{*}$ is number of relativistic degrees of freedom, $\langle\sigma v\rangle$ is the thermally averaged cross section for $S S$ annihilation into the SM particles (i.e. leptons and quarks , $f \bar{f}$, and gauge bosons, $W^{+} W^{-}, Z Z$, denoted collectively as $\left.X \bar{X}\right)$ and into Higgs bosons $\left(h h, h H, H H, A A, H^{+} H^{-}\right)$. The Feynman diagrams for all the processes are shown in 
figure 6. First, the process of annihilation into the SM particles is mediated by an $s$ channel $h$ or $H$ only. Following [38] (see also [43], which however has small numerical factor errors), we find

$$
\left\langle\sigma_{S S \rightarrow X \bar{X}} v_{\text {rel }}\right\rangle=\sum_{\mathcal{H}=h, H}\left|\frac{g_{\mathcal{H} S S} C_{X}^{\mathcal{H}}}{4 m_{S}^{2}-m_{\mathcal{H}}^{2}+i \Gamma_{\mathcal{H}} m_{\mathcal{H}}}\right|^{2} \frac{\Gamma_{\mathrm{SM}}\left(\mathcal{H}^{*} \rightarrow X \bar{X}\right)}{2 m_{S}}
$$

where $C_{X}^{\mathcal{H}}$ is the coupling of $\mathcal{H}$ to $X \bar{X}$ relative to the coupling of the SM Higgs boson to $X \bar{X}$ and $\Gamma_{\mathrm{SM}}\left(\mathcal{H}^{*} \rightarrow X \bar{X}\right)$ stands for the SM partial width in the $X \bar{X}$ final state calculated at invariant mass $\sqrt{s}=2 m_{S}$. (Note: for $X=Z$, then $\bar{X}=Z$ also. In this case, $\Gamma\left(\mathcal{H}^{*} \rightarrow X \bar{X}\right)$ must include the $1 / 2$ ! for identical particles in the final state.) In this equation, the total width, $\Gamma_{\mathcal{H}}$, must include the width for $\mathcal{H} \rightarrow S S$ and any partial width modifications relative to the SM width for the various SM channels (in particular, the enhancement of $\Gamma(H \rightarrow b \bar{b})$ at large $\tan \beta$ in the Type II case.)

Second, there are all the channels containing Higgs pairs. For the $\left(H_{i} H_{i}\right)=(A A)$ or $\left(H^{+} H^{-}\right)$final states, the relevant diagrams are the first two diagrams in the upper row of the figure, which include not only $s$-channel $h$ or $H$ exchange but also a four-point contact self-coupling. For final states containing CP-even Higgs pairs, $\left(H_{i} H_{j}\right)=(h h),(H H),(h H)$, there are contributions from $t$ - and $u$-channel $S$ exchange (the last two diagrams with different topologies in the top row of figure 6 ) in addition to the $s$-channel $h$ or $H$ exchange diagrams and the four-point contact self-coupling. A formula that applies to all these different cases is most easily given in terms of the Feynman rules for the various relevant vertices: the quartic Feynman rules were given earlier in eq. (3.11) and the trilinear coupling $g_{\mathcal{H} h H}$ Feynman rule can be found in appendix F of [61]. We find

$$
\begin{aligned}
\left\langle\sigma_{S S \rightarrow H_{i} H_{j}} v_{\mathrm{rel}}\right\rangle=\frac{\beta\left(m_{H_{i}}, m_{H_{j}}\right)}{32\left(1+\delta_{i j}\right) \pi m_{S}^{2}} \mid g_{H_{i} H_{j} S S} & \sum_{\mathcal{H}=h, H} \frac{g_{\mathcal{H} S S} g_{\mathcal{H} H_{i} H_{j}}}{4 m_{S}^{2}-m_{\mathcal{H}}^{2}+i \Gamma_{\mathcal{H}} m_{\mathcal{H}}} \\
& +\left.2 \delta_{C P} \frac{g_{H_{i} S S} g_{H_{j} S S}}{\frac{1}{2}\left(m_{H_{i}}^{2}+m_{H_{j}}^{2}\right)-2 m_{S}^{2}}\right|^{2}
\end{aligned}
$$

where

$$
\delta_{C P}= \begin{cases}0 & H_{i} H_{j}=A A, H^{+} H^{-} \\ 1 & H_{i} H_{j}=h h, H H, h H\end{cases}
$$

and

$$
\beta\left(m_{H_{i}}, m_{H_{j}}\right)=\left(1-\frac{m_{H_{i}}^{2}+m_{H_{j}}^{2}}{2 m_{S}^{2}}+\frac{\left(m_{H_{i}}^{2}-m_{H_{j}}^{2}\right)^{2}}{16 m_{S}^{4}}\right)^{1 / 2} .
$$

Note that some final states will typically be kinematically closed. In particular, for $m_{S}<$ $m_{h}$ only the $f \bar{f}(f \neq t), V V$ and, possibly, $A A$ channels will be allowed.

In order to illustrate results of the scan over singlet parameter space, in figures 7 and 8 we show $\Omega h^{2}$ as a function of $m_{S}$ for representative $2 \mathrm{HDM}$ points when scanning over the remaining singlet parameters. The $2 \mathrm{HDM}$ parameters for these four points are given in table 2. For the first case, figure 7 , the $2 \mathrm{HDM}$ parameters are such that low $m_{S}$ is eliminated 


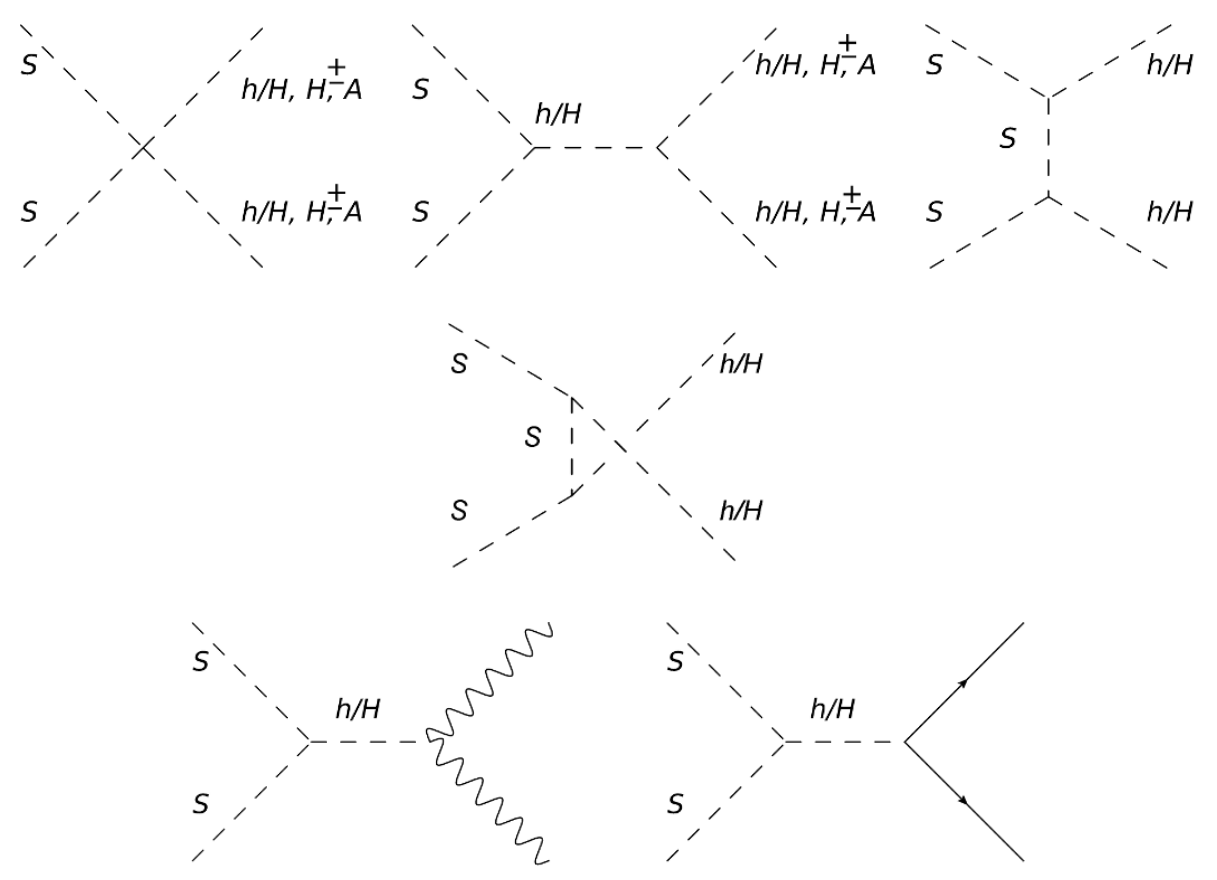

Figure 6. Singlet annihilation diagrams relevant for the relic density calculation.

\begin{tabular}{|c|c|c|c|c|c|c|c|c|c|c|c|c|}
\hline BMP \# & $\tan \beta$ & $\sin \alpha$ & $m_{12}^{2}$ & $m_{h}$ & $m_{H}$ & $m_{A}$ & $m_{H^{ \pm}}$ & $\lambda_{1}$ & $\lambda_{2}$ & $\lambda_{3}$ & $\lambda_{4}$ & $\lambda_{5}$ \\
\hline I-1 & 1.586 & -0.587 & 5621 & 123.71 & 534.25 & 645.13 & 549.25 & 5.98 & 1.683 & 3.203 & -1.032 & -4.81 \\
II-1 & 0.969 & -0.721 & $1.251 \times 10^{5}$ & 127.96 & 678.98 & 600.36 & 563.18 & 3.463 & 4.046 & -0.997 & -0.389 & -1.816 \\
\hline I-2 & 1.346 & -0.663 & -2236 & 126.49 & 168.01 & 560.92 & 556.94 & 1.199 & 0.59 & 10.101 & -5.12 & -5.267 \\
II-2 & 2.092 & -0.4096 & $-1.264 \times 10^{4}$ & 125.89 & 137.86 & 451.33 & 398.76 & 3.984 & 0.454 & 5.732 & -2.422 & -3.896 \\
\hline
\end{tabular}

Table 2. $2 \mathrm{HDM}$ parameters for the plots of figures 7 and 8 . Masses in $\mathrm{GeV} ; m_{12}^{2}$ in $\mathrm{GeV}^{2}$.

when correct EWSB is imposed in addition to stability and unitarity. In the second case, figure 8, a large range of $m_{S}$ values is consistent with EWSB and the observed $\Omega h^{2} \sim 0.1$. Note that for the case of figure $8, m_{H}$ is relatively small. This means that relatively modest values of $\left|\lambda_{H}\right|$ provide adequate annihilation for achieving the observed $\Omega h^{2}$. In contrast, in the case of figure 7 relatively large values of $m_{H}$ were employed. As a result, quite large values of $\left|\lambda_{H}\right|$ would be needed for sufficient annihilation. However, as shown in figure 5 , in the region of $m_{S} \leq 50 \mathrm{GeV} \mathrm{P}+\mathrm{S}+\mathrm{U}+\mathrm{EW}$ (especially the latter) require $\left|\lambda_{H}\right| \lesssim 3$, a value that is insufficient, implying that no points satisfying $\mathrm{P}+\mathrm{S}+\mathrm{U}+\mathrm{EW}$ (i.e. red points) are found in this region. In addition, at low $m_{S}$ values, it is possible that $\mathrm{BR}(h \rightarrow S S)$ is not below the $68 \%$ C.L. upper limit of $\sim 0.1$ required by fitting of the $h$ properties to the LHC data - see next subsection. The figures show the impact of the additional requirement of $\mathrm{BR}(h \rightarrow S S) \leq 0.1$.

In both figure 7 and figure 8, one can see a sharp dip in $\Omega h^{2}$ at $m_{S} \simeq 63 \mathrm{GeV}$ which arises from on-shell $h$ exchange, as well as a sudden drop in $\Omega h^{2}$ near $80 / 90 \mathrm{GeV}$ due to the $W W$ and $Z Z$ final states becoming available in the $S S$ annihilation (the relic abundance is inversely proportional to the annihilation cross section). A similar threshold appears 

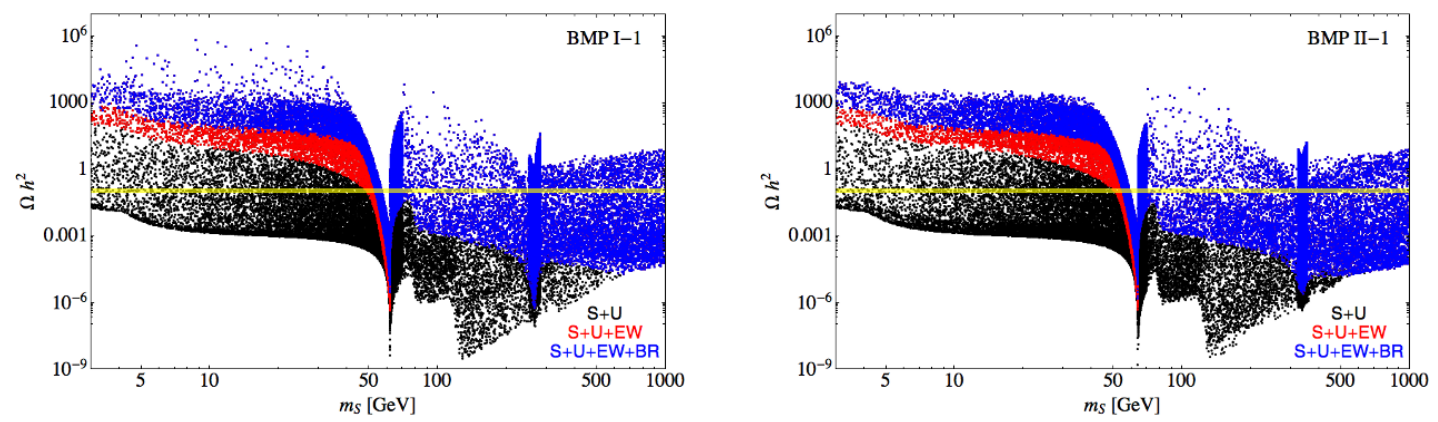

Figure 7. Results for the relic abundance $\Omega h^{2}$ as a function of $m_{S}$ coming from a scan over the singlet parameter space for a fixed $2 \mathrm{HDM}$ point. The sample $2 \mathrm{HDM}$ parameters employed are given in table 2. All points satisfy perturbativity as defined earlier. Black points satisfy the stability and unitarity conditions, red points satisfy also the EWSB conditions. Blue points satisfy $\mathrm{S}+\mathrm{U}+\mathrm{EW}$ and have $\mathrm{BR}(h \rightarrow S S) \leq 0.1$. The yellow band is the recent $\pm 3 \sigma$ Planck window $\Omega h^{2}=0.1187 \pm 0.0017$ at $68 \%$ CL [70]. We emphasize that the LUX and other limits on DM detection are not yet imposed in these plots.
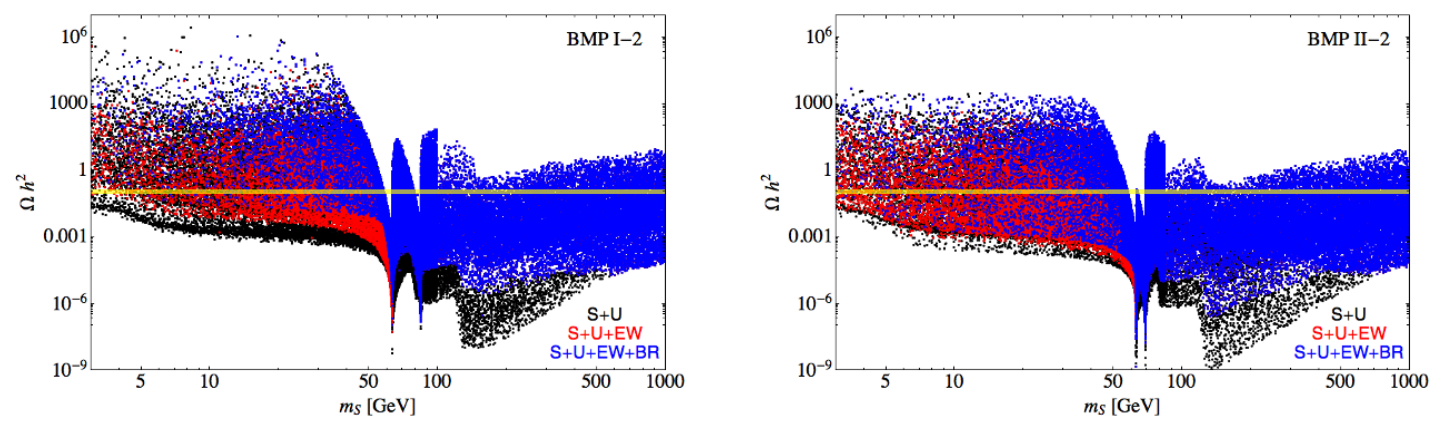

Figure 8. As for figure 7, but for different 2HDM points, see the last two points of table 2, chosen so that a large fraction of the low $m_{S}$ values pass all constraints other than limits on DM detection.

around $m_{S} \sim m_{t}$. One can also observe sharp dips in $\Omega h^{2}$, corresponding to $s$-channel exchange of the heavy scalar $H$, at $m_{S} \simeq m_{H} / 2 \sim 265 \mathrm{GeV}$ and $340 \mathrm{GeV}$ for Type I and Type II, respectively, for figure 7 and at $m_{S} \sim 85 \mathrm{GeV}$ and $68 \mathrm{GeV}$ in the case of figure 8 .

\subsection{Higgs invisible/unseen decays}

In addition to decays into SM particles, the CP-even Higgs bosons $h$ and $H$ of the 2HDMS have a number of possible invisible and/or "unseen" decays. By "unseen" we mean decay modes that contain visible particles, but that the experimental analyses have not explored and/or are not yet able to place useful limits on. The invisible decays are $h, H \rightarrow S S$ and the potentially important unseen decay modes are $h \rightarrow A A$ and $H \rightarrow A A, h h$. Since we assume that it is the $h$ that is the $\sim 125.5 \mathrm{GeV}$ state, we are not immediately concerned with $H$ decays. However, both $h \rightarrow S S$ and $h \rightarrow A A$ decays could make it impossible to fit the LHC Higgs data at the $68 \%$ C.L. level that we are requiring. In fact, at this level of fitting precision, the scans of [32] did not find points with $m_{A}<m_{h} / 2$. Thus, we 

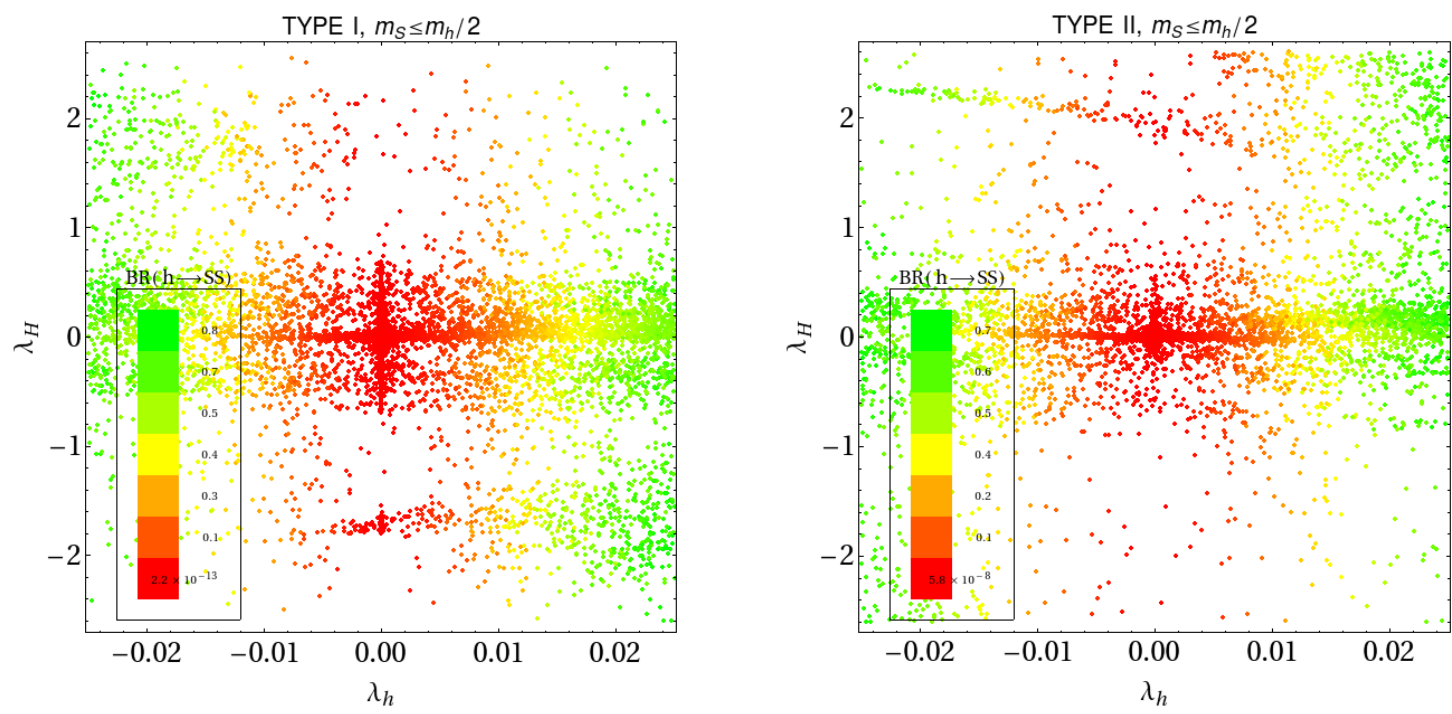

Figure 9. The couplings of $h / H$ to $S S$ after imposing the full set of constraints including $\Omega h^{2}$ but not LUX and without the $\mathrm{BR}(h \rightarrow S S) \leq 0.1$ constraint. Coloring is according to $\mathrm{BR}(h \rightarrow S S)$ : points with small BR are red, large BR points are green. The $2 \mathrm{HDM}$ points employed in this scan are the red points of figure 1. A full scan over the singlet sector parameters is performed subject to the standard $\mathrm{P}+\mathrm{S}+\mathrm{U}+\mathrm{EW}$ constraints.

need only ensure that, for each point in the full 2HDMS parameter space, $\operatorname{BR}(h \rightarrow S S)$ is sufficiently small as to not significantly disturb the fit of the $h$ to the LHC Higgs data. The $h, H \rightarrow S S$ decay widths are given by:

$$
\Gamma\left(h_{i} \rightarrow S S\right)=\frac{1}{2 \pi} \frac{g_{h_{i} S S}^{2}}{m_{h_{i}}} \sqrt{1-\frac{4 m_{S}^{2}}{m_{h_{i}}^{2}}}
$$

where $i=1,2$ denotes $h, H$ and the dimensional Feynman-rule couplings $g_{h_{i} S S}$ are given in eq. (3.11). In what follows, it will be most convenient to discuss results in the space of the dimensionless $\lambda_{H}$ vs. $\lambda_{h}$ parameters, where $g_{h_{i} S S}=-2 \lambda_{h_{i}} v$. When the decay $h \rightarrow S S$ is kinematically open, it will dominate the decay of the $h$ unless $\lambda_{h}$ is very small. Large $\mathrm{BR}(h \rightarrow S S)$ would invalidate the fits to the LHC $125.5 \mathrm{GeV}$ signal. The constraints on such an invisible decay are thus quite strong: $\mathrm{BR}(h \rightarrow S S) \leq 10 \%$ at $68 \%$ C.L. [60]. In practice, this bound is violated for most $m_{S}<55 \mathrm{GeV}$ points in the full 2HDMS parameter space leaving only a small number of points with $\lambda_{h} \ll 1$ for which $\mathrm{BR}(h \rightarrow S S) \leq 0.1$. This is illustrated in figure 9 , which shows points in the $\left(\lambda_{h}, \lambda_{H}\right)$ plane, coloured with respect to the resulting $B R(h \rightarrow S S)$. Invisible decays of the $H$ will be discussed later.

\subsection{Direct detection}

The rate at which DM-particles scattering off nuclei can be detected is directly related to the DM-nuclei scattering cross-section [71], which is given by:

$$
\sigma_{\mathrm{DM}-N}=\int_{0}^{4 \mu_{r}^{2} v^{2}} \frac{d \sigma(q=0)}{d|\mathbf{q}|^{2}} d|\mathbf{q}|^{2}=\frac{4 \mu_{r}^{2}}{\pi} f_{p}^{2}\left[Z+\frac{f_{n}}{f_{p}}(A-Z)\right]^{2}
$$




\begin{tabular}{|c|c|c|c|}
\hline$q$ & $u$ & $d$ & $s$ \\
\hline$f_{T q}^{p}$ & 0.0153 & 0.0191 & 0.0447 \\
\hline$f_{T q}^{n}$ & 0.0110 & 0.0273 & 0.0447 \\
\hline
\end{tabular}

Table 3. Form factors extracted from micrOMEGAs 3.0.

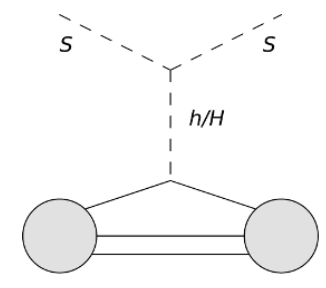

Figure 10. Feynman diagram for the scattering of DM off a nucleon.

where $\mathbf{q}$ is the momentum transfer, $\mu_{r}=\left(m_{N} m_{S}\right) /\left(m_{N}+m_{S}\right)$ and $v$ is the relative velocity. The couplings of DM to the proton and neutron, $f_{p}$ and $f_{n}$, can be expressed as

$f_{N}=\frac{m_{N}}{2 m_{S}}\left(\sum_{q=u, d, s} f_{T q}^{N} \frac{\lambda_{S S q q}}{m_{q}}+\frac{2}{27} f_{T G}^{N} \sum_{q=c, b, t} \frac{\lambda_{S S q q}}{m_{q}}\right), f_{T G}^{N}=1-\sum_{q=u, d, s} f_{T q}^{N}, \quad(N=p, n)$.

where $m_{N}$ is the mass of the nucleon, $f_{T q}^{N}$ is the form factor of the nucleon (see table 3 ) and $\lambda_{S S q q}$ is the effective coupling of the DM particle $S$ to a $q$-flavor quark component in the nucleon. In the 2HDMS, this interaction derives from $t$-channel exchange of the $h$ and $H$, as illustrated in figure 10. Thus, in the limit of zero momentum transfer, the Higgs $h_{i}=h$ or $H$ propagator reduces to $\frac{i}{-m_{h_{i}}^{2}}$ and we find

$$
\lambda_{S S q q}=\sum_{h_{i}=h, H} \frac{g_{h_{i} S S} g_{h_{i} q \bar{q}}}{-m_{h_{i}}^{2}}=\left(\frac{2 \lambda_{h}}{m_{h}^{2}} C_{q}^{h}+\frac{2 \lambda_{H}}{m_{H}^{2}} C_{q}^{H}\right) m_{q}
$$

where we have used $g_{h_{i} q \bar{q}}=-i \frac{g m_{q}}{2 m_{W}} C_{q}^{h_{i}}\left(m_{W}=\frac{1}{2} g v\right.$ in our convention) with the quark coupling factors $C_{q}^{h_{i}}$ for Type I and II models as listed in table 1 and the Feynman rule $g_{h_{i} S S}$ expressions given in eq. (3.11). In practice, direct detection rates in our calculation have been evaluated using micrOMEGAs [72], including QCD NLO corrections.

There are numerous collaborations (LUX, XENON 100, SuperCDMS, CDMS, CoGeNT and DAMA being of particular interest to us) working on the direct detection of DM. They typically translate the limit on the event rate against recoil energy they directly detect into a limit on the DM-proton cross section $\sigma_{\mathrm{DM}-p}$ as a function of DM mass. However, in reality there are several standard assumptions hidden in this translation that might or might not be correct. For instance, they assume a DM halo in the vicinity of Earth and employ the truncated Maxwell-Boltzmann velocity distribution below the escape velocity obtained from the Standard Halo Model. They also assume that the DM particle elastically scatters with a short range contact interaction via a 'heavy mediator', implying zero-momentum 
transfer. Of particular importance, they adopt the assumption that DM has equal coupling to the neutron and proton, that is to say the ratio $f_{n} / f_{p}=1$.

Indeed, this equality approximately holds in the Type I model because of the universal coupling structure with up-type and down-type quarks, see table 1 . In fact, $\frac{\lambda_{S S q q}}{m_{q}}$ is independent of quark-species and the common couplings $C_{U, D}^{h}$ and $C_{U, D}^{H}$ in the Type I model can be factored out and will then cancel out in the ratio. From eqs. (4.8) and (4.9), one can then derive the ratio of $f_{n} / f_{p}$ in the Type I case:

$$
\frac{f_{n}}{f_{p}}=\frac{m_{n}}{m_{p}} \frac{\sum_{q=u, d, s} f_{T q}^{n}+\frac{2}{27} f_{T G}^{n} \sum_{q=c, b, t}}{\sum_{q=u, d, s} f_{T q}^{p}+\frac{2}{27} f_{T G}^{p} \sum_{q=c, b, t}} \approx 1.01208
$$

This result implies that isospin-violating effects for DM-nucleon scattering are negligible for a Type I 2HDMS and that one can thus directly compare results of our calculations with all experimental bounds including the LUX and SuperCDMS upper limits and the CDMS II/CRESST positive signals.

However, the relation $f_{n} / f_{p}=1$ is not always true in the Type II model. In order to compare the predicted cross-sections for DM-nucleon scattering with the results presented by the experimental groups, we define the nucleon-normalized cross section, $\bar{\sigma}_{\mathrm{DM}-p}$, following $[10,12]$ :

$$
\bar{\sigma}_{\mathrm{DM}-p}=\sigma_{\mathrm{DM}-p} \Theta_{X}\left(f_{n}, f_{p}\right)
$$

where $\sigma_{\mathrm{DM}-p}$ is the predicted DM-proton cross-section and the rescaling factor $\Theta_{X}$ is defined as

$$
\Theta_{X}\left(f_{n}, f_{p}\right) \equiv \begin{cases}{\left[\frac{Z}{A}+\frac{f_{n}}{f_{p}}\left(1-\frac{Z}{A}\right)\right]^{2},} & \text { single isotope detector } \\ \frac{\sum_{I} \eta_{I} \mu_{A_{I}}^{2}\left[Z+f_{n} / f_{p}\left(A_{I}-Z\right)\right]^{2}}{\sum_{I} \eta_{I} \mu_{A_{I}}^{2} A_{I}^{2}}, & \text { multiple isotope detector }\end{cases}
$$

where $I$ runs over all isotopes present in the detector $X$ and $\eta_{I}$ is the relative abundance of the $I$ 'th isotope. Note that if $f_{n} / f_{p}=1$, then $\Theta_{X}\left(f_{n}, f_{p}\right)=1$. However, when $f_{n} / f_{p} \neq 1, \Theta_{X}\left(f_{n}, f_{p}\right)$ will depend upon the isotope abundances and is therefore determined by the properties of the chemical elements used in the various detectors. It was pointed out in [11-14] that the scattering amplitudes of DM with proton and neutron may interfere destructively in such a way as to achieve $f_{n} / f_{p} \sim-0.7$, the value for which the resulting LUX exclusion limits are not in strong conflict with the favored signal regions of the Silicon-based CDMS II experiment and the Germanium-based CoGeNT experiment. However, these positive signal regions are in direct conflict with the limits obtained by SuperCDMS $[15,16]$. In any case, in order to interpret any given DM scattering result, it is necessary to compute $f_{n} / f_{p}$ for each Type II parameter point. Further, $f_{n} / f_{p}$ in general depends on the singlet sector parameters.

However, there is an interesting special case in which $f_{n} / f_{p}$ depends only on the $2 \mathrm{HDM}$ parameters. Recalling that the positive CDMS II and CoGeNT signals are both at rather low $m_{S} \sim 6-12 \mathrm{GeV}$ and noting that $B R(h \rightarrow S S)$ will be large for such masses unless 

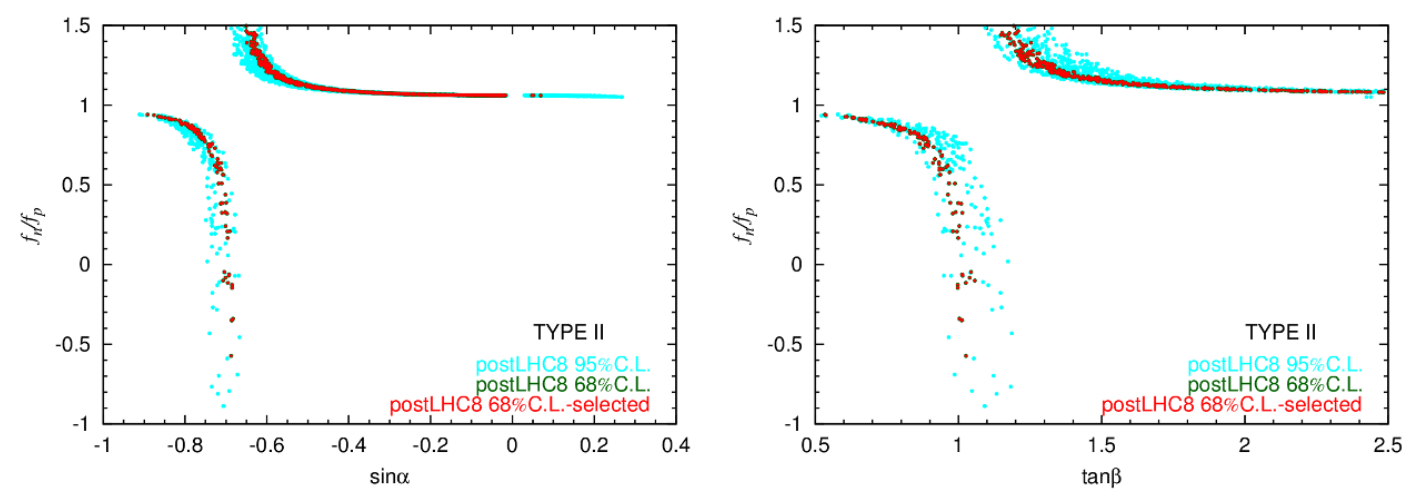

Figure 11. $f_{n} / f_{p}$ in the limit of $\lambda_{h}=0$ as a function of $\sin \alpha$ and $\tan \beta$ for the $68 \%$ C.L. Type II 2HDM scan points.

$\lambda_{h}$ is very small, it is useful to give an approximation for $f_{n} / f_{p}$ in the limit of $\lambda_{h} \rightarrow 0$, i.e. in the limit of ignoring the $h$ term in eq. (4.8). In this limit, the value of $f_{N}$ depends only on the quark couplings of the $H$ :

$$
\begin{aligned}
f_{N}=\frac{m_{N}}{2 m_{S} m_{H}^{2}}\left\{\left[f_{T u}^{N}+\frac{2}{27}\left(f_{T G c}^{N}\right.\right.\right. & \left.\left.+f_{T G t}^{N}\right)\right] C_{u}^{H} \\
& \left.+\left(f_{T d}^{N}+f_{T s}^{N}+\frac{2}{27} f_{T G b}^{N}\right) C_{d}^{H}\right\}, \quad(N=p, n) .
\end{aligned}
$$

In figure 11, we display the resulting $f_{n} / f_{p}$ as a function of $\sin \alpha$ for the Type II points from [32] that give Higgs boson property fits at the 95\% C.L. or better. There, we see a large range of $f_{n} / f_{p}$ values, ranging from +1.5 to $\sim-0.9$. However, for the $68 \%$ C.L. Type II points that we include in our study, points with substantially negative $f_{n} / f_{p}$ are rather sparse, with the most negative value associated with a single isolated point close to -0.7 . This is just an accidental result given the scanning procedure/density employed in [32].

The rather singular structure of this plot can be understood as follows. Since the LHC data at $68 \%$ C.L. are in good agreement with SM predictions, most of the Type II $2 \mathrm{HDM}$ points shown in figure 1 have $\beta-\alpha \simeq \pi / 2$, in which case $C_{u}^{H} \simeq-\cot \beta, C_{d}^{H} \simeq \tan \beta$ in the Type II model. In this approximation, one can use eq. (4.13) to obtain $\tan \beta$ as a function of $f_{n} / f_{p}$ in the limit of $\lambda_{h} \rightarrow 0$ :

$$
\tan ^{2} \beta\left(f_{n} / f_{p}\right)=\frac{\frac{f_{n}}{f_{p}} F_{u}^{p}-\frac{m_{n}}{m_{p}} F_{u}^{n}}{\frac{f_{n}}{f_{p}} F_{d}^{p}-\frac{m_{n}}{m_{p}} F_{d}^{n}}
$$

where

$$
F_{u}^{N} \equiv f_{T u}^{N}+\frac{2}{27}\left(f_{T G c}^{N}+f_{T G t}^{N}\right), \quad F_{d}^{N} \equiv f_{T d}^{N}+f_{T s}^{N}+\frac{2}{27} f_{T G b}^{N} .
$$

For the value $f_{n} / f_{p}=-0.7$, one finds $\tan \beta=1.04364$ implying $\alpha \simeq-\frac{\pi}{4}$ and $\sin \alpha \sim$ -0.707 , with a small variation associated with the exact form factor values. Although we have a single point with these approximate values, it turns out that for $\mathrm{BR}(h \rightarrow S S) \leq 0.1$ 
the possible $\Omega h^{2}$ values lie outside the $3 \sigma$ window that we have allowed. Thus, within the limitations of the scanning so far performed we have not managed to produce a point that satisfies all the constraints not related to DM-scattering that also has $f_{n} / f_{p} \sim-0.7$, but we regard it as possible that much denser scans might reveal a point of this type. Of course, to the extent that we accept the SuperCDMS upper bound, the CDMS II result is excluded in any case given that all the acceptable points have $f_{n} / f_{p}$ values that are close to 1 . So, it is perhaps a good feature of the 2HDMS model that obtaining a point consistent with $f_{n} / f_{p} \sim-0.7$ and all other constraints requires a very fine-tuned choice of $\tan \beta$ and $\sin \alpha$.

\section{DM full mass scan}

As noted earlier, instead of scanning over the full 2HDMS parameter space, for simplicity we used selected points from the 2HDM phenomenologically allowed points of [32] (labelled as "postLHC8-FDOK"), as outlined in section 2. In the case of $m_{h} \sim 125.5 \mathrm{GeV}$, the $2 \mathrm{HDM}$ analysis of [32] found $\sim 5200$ points consistent with Higgs observations at $68 \%$ C.L. in the Type I model, from which we randomly chose 1250 points for further analysis. For the Type II model we use all of the $\sim 900$ points that fall within the $68 \%$ C.L. criterion. These points are marked in red in figure 1 . For each surviving $2 \mathrm{HDM}$ point, we perform a scan over the extra singlet parameters: $m_{S}, \lambda_{h}, \lambda_{H}$. We then check theoretical constraints for the 2HDMS model including perturbativity, stability, unitarity and proper electroweak symmetry breaking, as discussed in section 3 . Since the extra scalar $S$ does not acquire a VEV, it does not mix with the other Higgs bosons $h$ and $H$. As a result, the experimental constraints from electroweak precision tests (STU parameters), $B$ physics, direct searches at LEP and also limits on the heavier Higgs bosons ( $H$ and possibly $A$ ) are barely influenced by the presence of the singlet scalar $S$. Therefore, the postLHC8-FDOK points in the $2 \mathrm{HDM}$ can be adopted as good starting points when expanding to the 2HDMS. As we have noted, the only caveat that arises is the need to take into account the possibility of $h \rightarrow S S$ decays when the scalar $S$ is light. Substantial $\operatorname{BR}(h \rightarrow S S)$ will spoil the pure 2HDM fit performed in [32]. Including limits from the current Higgs invisible decay searches at the LHC one finds roughly that $\mathrm{BR}(h \rightarrow S S) \leq 30 \%(10 \%)$ is required at $95 \%(68 \%)$ C.L. Therefore, as discussed earlier, we impose a cut of $B R(h \rightarrow S S) \leq 10 \%$ for all points presented in the following context (except for a few situations as described later) in order to maintain the LHC signal fit and consistency with invisible decay limits. Finally, we use micrOMEGAs [72] to calculate the relic abundance of the DM candidate $S$ and require that the predicted $\Omega h^{2}$ fall within the $\pm 3 \sigma$ Planck window $\Omega_{\mathrm{DM}}^{\exp } h^{2}=0.1187 \pm 0.0017$ at $68 \%$ C.L. [70]. Hereafter, we refer to this set of constraints as the "preLUX" constraints.

Let us now turn to the issue of DM scattering on nuclei. For the points satisfying the "preLUX" constraints, we calculate the cross section for the scattering of the $S$ off a nucleon and compare the predicted value $\bar{\sigma}_{\mathrm{DM}-p}$ (after rescaling by $\Theta$ in the case of Type II) to the latest LUX limits for the DM-proton cross section, denoted $\sigma_{\mathrm{DM}-p}^{\mathrm{LUX}}$ (which are obtained assuming $f_{n} / f_{p}=1$ ). If the points obey the condition $\bar{\sigma}_{\mathrm{DM}-p} \leq \sigma_{\mathrm{DM}-p}^{\mathrm{LUX}}$, they are not excluded by the LUX limit. 


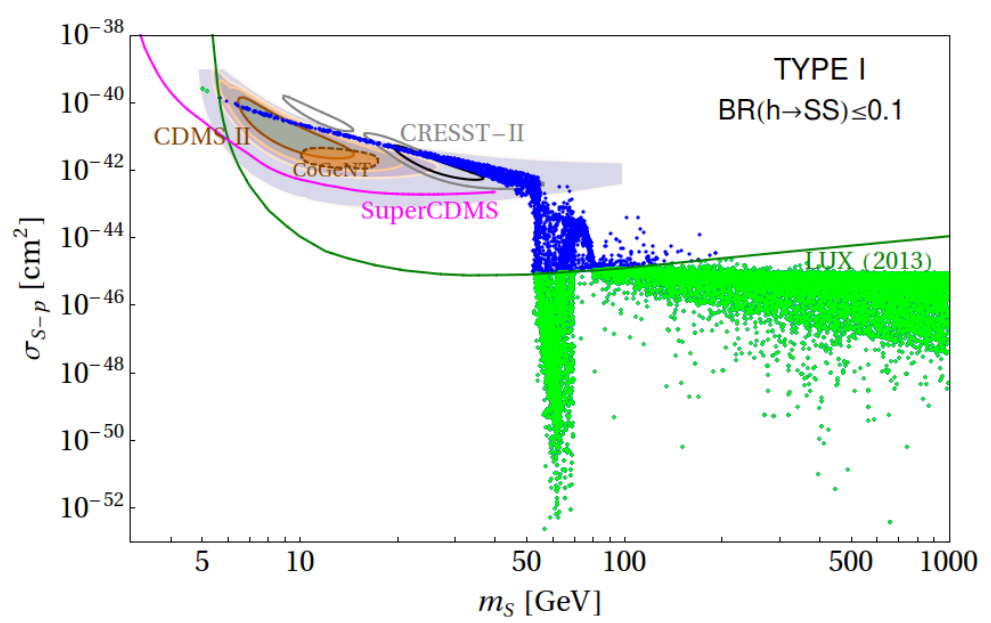

Figure 12. Cross section for DM-proton scattering for the Type I model. All points shown satisfy the full set of preLUX constraints, including $\operatorname{BR}(h \rightarrow S S) \leq 0.1$, while the green points satisfy in addition the LUX limits. The pink and green lines are the limits from SuperCDMS and LUX, respectively. Recall that for Type I, $f_{n} / f_{p} \sim 1$ and so no rescaling is required between target types. Also shown are contours corresponding to the CRESST-II, CoGeNT and CDMS II positive signal regions. In the case of CRESST-II, the darker black contour is at $68 \%$ C.L. and the lighter grey contours are at $95 \%$ C.L. In the case of CoGeNT (orange region) we show only the $90 \%$ C.L. contour. For CDMS II, we display contours (using various levels of grey) at $68 \%, 90 \%, 95 \%$ and $99 \%$ C.L.

\subsection{Type I analysis}

In figure 12 we present the cross section versus $m_{S}$ for the Type I model. Since $f_{n} / f_{p} \sim 1$ in the case of the Type I model, all experimental results can be displayed on the same plot. Points obeying the LUX limit are shown in green. Points that do not pass the LUX limit but do satisfy all preLUX conditions (including correct $\Omega h^{2}$ and $\operatorname{BR}(h \rightarrow S S) \leq 0.1$ ) are shown in blue. Note that few green points at very low $m_{S}$ that pass the LUX limit are excluded by the SuperCDMS limit. Note that the Type I predictions for $\bar{\sigma}_{\mathrm{DM}-p}$ agree pretty well with CDMS II/CRESST-II data (for more detailed discussion, see section 5.3.1, but, of course, disobey the LUX limit. The narrowness of the $\bar{\sigma}_{\mathrm{DM}-p}$ band at low $m_{S}$ can be understood as follows. In this mass region, we know that $\lambda_{h} \simeq 0, \mathrm{DM}$ annihilation and scattering off nucleons are thus realized via $H$ exchange in the $s$ - and $t$-channels, respectively. Both processes are essentially controlled by the ratio $\lambda_{H} / m_{H}^{2}$. We observe that once the constraints of $B R(h \rightarrow S S) \leq 10 \%$ and good $\Omega h^{2}$ are both satisfied $\lambda_{h}$ and $\lambda_{H}$ are roughly fixed. As a result, the predicted value of $\sigma_{S-p}$ as a function of $m_{S}$ is constrained to a narrow band that happens to pass through the CDMS II/CRESST-II preferred regions. However, the CDMS II/CRESST-II regions are simply not consistent with the combination of LUX and SuperCDMS limits in the Type I model. Finally, once $m_{S} \gtrsim 55 \mathrm{GeV}$ essentially all of the points that are consistent with preLUX constraints also pass the LUX limit (SuperCDMS limits do not extend to masses $\gtrsim 40 \mathrm{GeV}$ ).

In figure 13, we display the associated $\lambda_{h}$ and $\lambda_{H}$ values as a function of $m_{S}$. We see that for $m_{S} \lesssim 50 \mathrm{GeV}$, the restriction to small $\lambda_{h}$ coming from requiring $\mathrm{BR}(h \rightarrow S S) \leq 0.1$ 

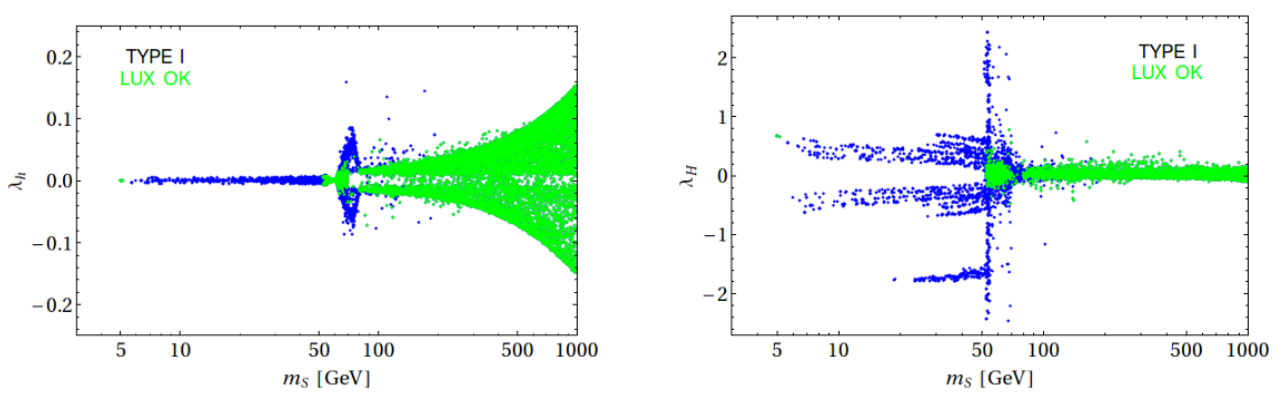

Figure 13. The couplings $\lambda_{h}$ and $\lambda_{H}$ as a function of $m_{S}$ for Type I. All points shown satisfy the full set of preLUX constraints; blue points are excluded by LUX while green points are allowed by LUX results. The green points at very low $m_{S}$ are, however, excluded by SuperCDMS.

implies that $S S \rightarrow H \rightarrow S M$ will be dominant and correct $\Omega h^{2}$ then requires relatively substantial $\lambda_{H}$, the precise value depending on $m_{H}$, see eq. (4.2). In contrast, there is a considerable variety of possibilities for $\lambda_{h}$ and $\lambda_{H}$ in the "resonance" region, i.e. in the vicinity of $m_{S} \sim m_{h} / 2$. Typically, both the $h$ and $H s$-channel diagrams contribute to $S S \rightarrow X \bar{X}$. Once $m_{S}$ is above the resonance region, many channels open up and $\lambda_{h}$ is no longer restricted by a limit on $\mathrm{BR}(h \rightarrow S S)$. A significant range of $\lambda_{h}$ becomes possible, the larger the value of $m_{S}$ the larger the range. Note that only in the resonance region are large values of $\lambda_{H}$ possible. There, contributions from $h$ and $H$ exchange can partially cancel. The fact that neither $\lambda_{h}$ nor $\lambda_{H}$ can be very large above the resonance region reflects the large number of final states that become available, in particular the $h h$ channel opens up once $m_{S} \gtrsim m_{h}$.

We note that the "band" structure in the $\lambda_{H}$ vs. $m_{S}$ plot in the $m_{S} \lesssim 50 \mathrm{GeV}$ region is due to the fact that $H$ exchange is dominant for $S S \rightarrow X \bar{X}$ annihilation. One finds that each band is associated with a particular $m_{H}$ value for the associated 2HDM point. As expected from eq. (4.2), the larger the value of $m_{H}$ the larger the value of $\lambda_{H}$ that is needed for correct $\Omega h^{2}$.

\subsection{Type II analysis}

We now turn to the Type II model. A particularly interesting question is whether or not one can have consistency between the CDMS II/CRESST-II preferred regions and the LUX limits. As already noted, this requires $f_{n} / f_{p} \sim-0.7$. As a first step, we examine the correlation between the ratio of $f_{n} / f_{p}$ and $\mathrm{BR}(h \rightarrow S S)$, as illustrated in figure 14. After imposing the constraint $\mathrm{BR}(h \rightarrow S S) \leq 10 \%$, as well as all the other preLUX constraints, all points with $f_{n} / f_{p} \sim-0.7$ in the low $m_{S}$ region are excluded. Indeed, in the low $m_{S}$ region $f_{n} / f_{p} \gtrsim 1$. Even relaxing the invisible decay limit to $B R(h \rightarrow S S) \leq 55 \%$ (the most conservative upper bound on $\mathrm{BR}_{\text {inv }}$ at the LHC [73]) still does not allow for points with $f_{n} / f_{p} \sim-0.7$. In the resonance region of $m_{S} \simeq 55 \mathrm{GeV}$, a predicted $f_{n} / f_{p}$ values range from below -1 to above 2 , although the majority of points have $f_{n} / f_{p}$ near 1 . Above the resonance region, i.e. $m_{S} \gtrsim m_{h} / 2$, most points have $f_{n} / f_{p} \sim 1$, but there is a handful of points with $f_{n} / f_{p}$ values both substantially above 1 and substantially below 1 (a few 


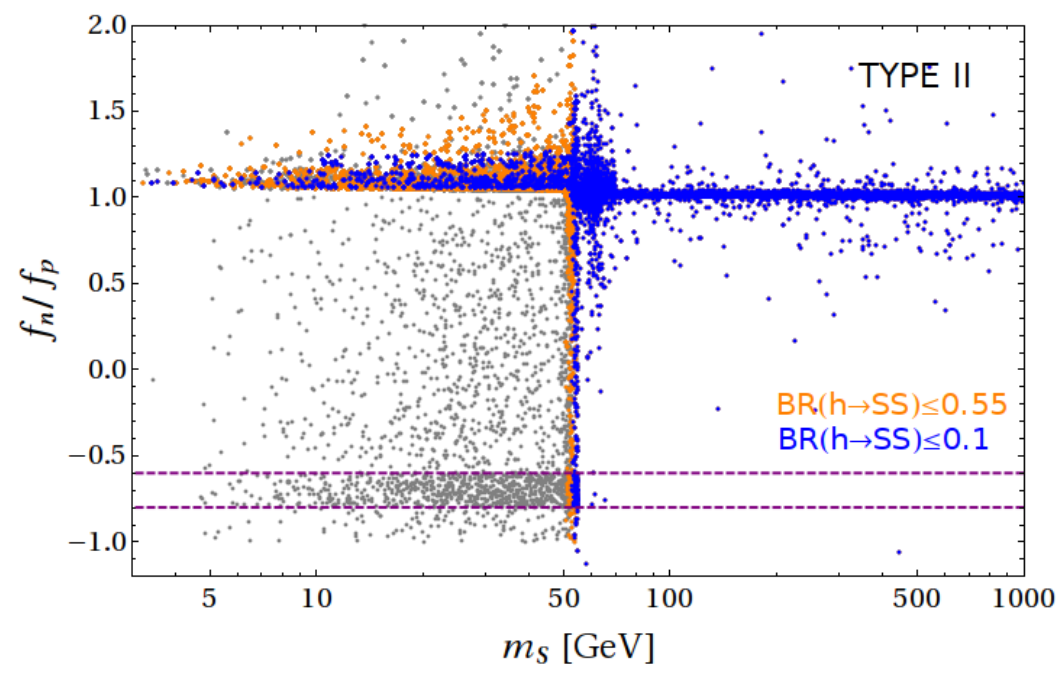

Figure 14. We display the correlation between the ratio of $f_{n} / f_{p}$ and $\mathrm{BR}(h \rightarrow S S)$ for points that obey all the preLUX constraints (i.e. theoretical constraints for 2HDMS, 2HDM fitting and correct $\left.\Omega_{D M}\right)$. Blue points have $\mathrm{BR}(h \rightarrow S S) \leq 0.1$ (therefore blue points satisfy the full set of preLUX constraints). The purple dashed line is located at $f_{n} / f_{p}=-0.7 \pm 0.1$. Most grey points have $\operatorname{BR}(h \rightarrow S S) \simeq 1$.

points have quite negataive values). Thus, in our predictions for DM scattering, it will be important to take into account the variation of $f_{n} / f_{p}$.

In order to present the overall picture for Type II, we adopt the parameters in [10] to calculate the rescaling factor $\Theta_{\mathrm{Xe}}$ for the Xenon-based detectors and present the $\bar{\sigma}_{D M-p}$ cross sections in figure 15. In the left plot, we impose all preLUX constraints (including $\Omega h^{2}$ in the $3 \sigma$ window) other than $\operatorname{BR}(h \rightarrow S S) \leq 0.1$. Points with $f_{n} / f_{p} \sim 1$ (for which $\left.\Theta_{\mathrm{Xe}} \sim 1\right)$ are singled out as are points with $f_{n} / f_{p} \sim-0.7$. Comparing with the right plot, one can find that only the former points can have $\mathrm{BR}(h \rightarrow S S) \leq 0.1$, and only a subset of these can obey the LUX limits. Basically, we find that obtaining correct $\Omega h^{2}$ while at the same time having $\mathrm{BR}(h \rightarrow S S) \leq 0.1$ (or even $\leq 0.55$ ) is not possible for the $f_{n} / f_{p} \sim-0.7$ points in the low- $m_{S}$ region.

To explore in more detail the level of inconsistency between the LUX and SuperCDMS limits and the positive signal regions for CDMS II and CoGeNT, we present figure 16 which focuses on the $m_{S} \leq 35 \mathrm{GeV}$ mass range. All plotted points obey the full set of preLUX constraints (including $\mathrm{BR}(h \rightarrow S S) \leq 0.1$ ). For the left figure, we have rescaled the DM-proton scattering cross section predicted for a given point by the factor $\Theta_{X}$, see eq. (4.12), as computed for $\mathrm{X}=\mathrm{Si}$ in order to compare to the positive signal region found by the CDMS II Silicon detector. We also display the relevant limits from the SuperCDMS experiment. These are $f_{n} / f_{p}$ dependent. The two lines correspond to the SuperCDMS limit after rescaling from the SuperCDMS Germanium target to the CDMS-II Silicon target. We rescaled $\sigma_{\mathrm{Si}}^{\text {SuperCDMS }}=\sigma^{\text {SuperCDMS }} \Theta_{\mathrm{Si}}\left(f_{n}, f_{p}\right) / \Theta_{\mathrm{Ge}}\left(f_{n}, f_{p}\right)$ using $f_{n} / f_{p}=1.05$ and $1.25-$ the minimum and maximum values shown in figure 14 for $m_{S} \leq 35 \mathrm{GeV}$ when $\operatorname{BR}(h \rightarrow$ $S S) \leq 0.1$ is imposed. We see that for the predicted range of $f_{n} / f_{p}$ the resulting rescaling 

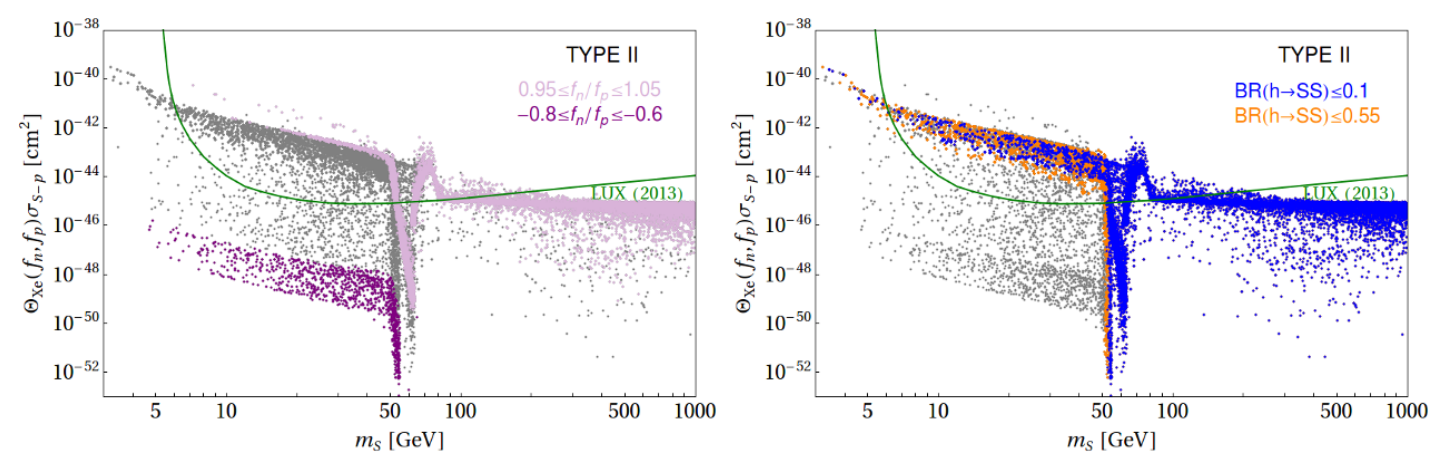

Figure 15. Cross section for DM - proton scattering for the Type II model rescaled by the function $\Theta_{\mathrm{X}}$ defined in eq. (4.12), where $\mathrm{X}=\mathrm{Xe}$ for a Xenon-based detector. All points plotted satisfy the preLUX constraints except $\operatorname{BR}(h \rightarrow S S) \leq 0.1$ (i.e. they satisfy the theoretical constraints for 2HDMS, 2 HDM fitting at $68 \%$ C.L. and the constraint on $\Omega h^{2}$ ). In the left-hand plot, for the light purple points the ratio $f_{n} / f_{p}$ is within the range $(0.95,1.05)$. For the darker purple points $-0.8 \leq f_{n} / f_{p} \leq-0.6$. The right-hand plot displays points that obey $\operatorname{BR}(h \rightarrow S S) \leq 0.1$ in blue (i.e. they obey the full set of preLUX constraints), while the orange points obey only the weaker limit of $\mathrm{BR}(h \rightarrow S S) \leq 0.55$.
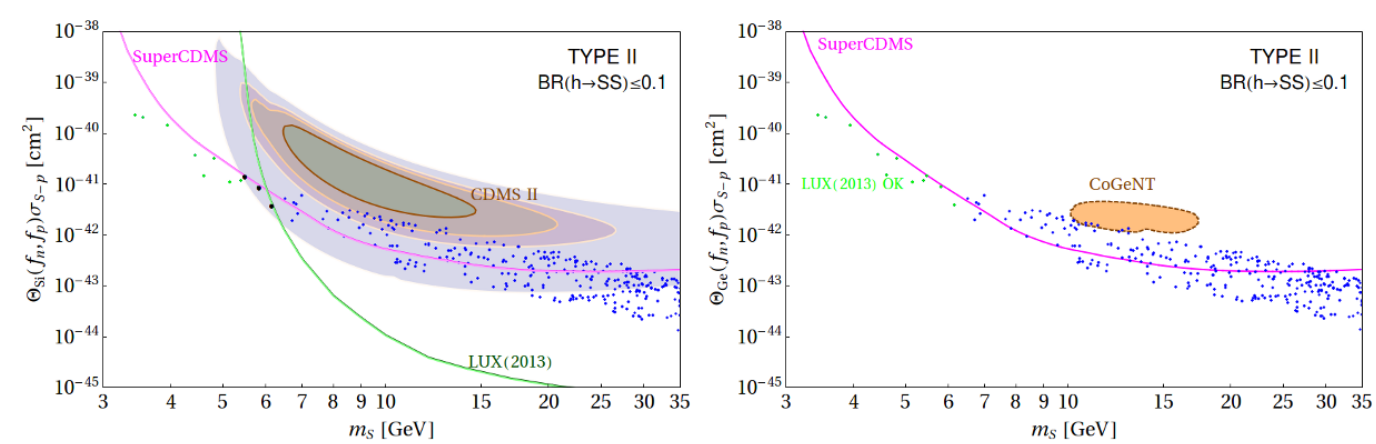

Figure 16. Cross section for DM-proton scattering for the Type II model rescaled by the function $\Theta_{\mathrm{X}}$ defined in eq. (4.12), where $\mathrm{X}=\mathrm{Si}$ for a Silicon detector (CDMS II) on the left and $\mathrm{X}=\mathrm{Ge}$ for the Germanium detector (CoGeNT) on the right. All points satisfy all the preLUX constraints (i.e. they satisfy the theoretical constraints for $2 \mathrm{HDMS}, 2 \mathrm{HDM}$ fitting at $68 \%$ C.L., $\mathrm{BR}(h \rightarrow S S) \leq 0.1$ and the constraint on $\left.\Omega h^{2}\right)$. The CDMS II contours shown are at $68 \%, 90 \%, 95 \%$ and $99 \%$ C.L. The CoGeNT contour is the $90 \%$ C.L. level contour. Light green points are allowed by LUX results. The larger black points are those allowed by both SuperCDMS and LUX and that also lie within the $99 \%$ C.L. CDMS II contour. The pink and light pink lines (almost degenerate) correspond to the SuperCDMS limit, after rescaling from the SuperCDMS Germanium target to the CDMS-II Silicon target using $f_{n} / f_{p}=1.05$ and 1.25 (the minimum and maximum values shown in figure 14 for $\operatorname{BR}(h \rightarrow S S) \leq 0.1$ when $\left.m_{S} \leq 35 \mathrm{GeV}\right)$. Also shown by the dark green lines is the rescaled LUX limit, $\sigma_{\mathrm{Si}}^{\mathrm{LUX}}=\sigma^{\mathrm{LUX}} \Theta_{\mathrm{Si}}\left(f_{n}, f_{p}\right) / \Theta_{\mathrm{Xe}}\left(f_{n}, f_{p}\right)$, using the same two $f_{n} / f_{p}$ values. 
is fairly minimal and those two limits are almost degenerate. Also shown by another two lines is the rescaled LUX limit, $\sigma_{\mathrm{Si}}^{\mathrm{LUX}}=\sigma^{\mathrm{LUX}} \Theta_{\mathrm{Si}}\left(f_{n}, f_{p}\right) / \Theta_{\mathrm{Xe}}\left(f_{n}, f_{p}\right)$, using the same two $f_{n} / f_{p}$ values. From this plot, we observe that there are a few points (the large black points) with $m_{S} \sim 5.5-6.2 \mathrm{GeV}$ that lie below both the rescaled LUX limits and rescaled SuperCDMS limits. Further, although these points lie below the $2 \sigma$ (95\% C.L.) contour of the positive signal region of CDMS II, they do fall within the $3 \sigma$ ( $99 \%$ C.L.) contour. Thus, the 2HDMS Type II model allows consistency between the CDMS II signal region (at 99\% C.L.) and the SuperCDMS and LUX limits for a small range of low $m_{S}$.

It is perhaps important to understand the points in figure 16 with low $m_{S}$ that obey LUX and SuperCDMS constraints in the case of the Type II model. Their properties appear in table 4 . All have low $\tan \beta$, very modest $m_{H}$ with $m_{A}, m_{H^{ \pm}}$somewhat larger (in the $300-600 \mathrm{GeV}$ range). It is worth recalling that for each $2 \mathrm{HDM}$ phenomenologically allowed point, the $2 \mathrm{HDM}$ parameters including $\tan \beta, \sin \alpha, m_{h}, m_{H}, m_{A}, m_{H^{ \pm}}$and $m_{12}$ are fixed. We then randomly scan over the singlet sector parameters $\kappa_{1}, \kappa_{2}$ (or equivalently $\left.\lambda_{h}, \lambda_{H}\right)$ and $m_{S}$. Therefore, one can have many values of $m_{S}$ and corresponding rescaled cross section (the pair of numbers appearing in the last column of table IV) for each fixed $2 \mathrm{HDM}$ point whose parameters are listed in the first 6 columns.

For the right figure, we rescale $\sigma_{\mathrm{DM}-p}$ using $\Theta_{X}$ as computed for $\mathrm{X}=\mathrm{Ge}$ in order to compare to the potential signal region for the CoGeNT Germanium detector. We find points consistent with all pre-LUX constraints within the CoGeNT 90\% C.L. signal region for $m_{S} \sim 10-15 \mathrm{GeV}$. However, the entire CoGeNT signal region is excluded by the SuperCDMS limit (no relative rescaling required since both are for a Germanium target) and by the LUX limit as indicated by the point coloring (where these limits have been rescaled using the $f_{n} / f_{p}$ value for a given point to determine whether or not the point is excluded).

In the case of both the CDMS II figure and the CoGeNT figure, we note that allowing $\mathrm{BR}(h \rightarrow S S)$ larger than 0.1 does not allow points much above those already shown, but rather increases the density of points where points are already shown.

As in the case of Type I, we could plot $\lambda_{h}$ and $\lambda_{H}$ vs. $m_{S}$ for the Type II points that obey preLUX constraints. The resulting point distributions look very similar to those shown in figure 13.

\subsection{Summaries}

It is perhaps useful to summarize what Type I and II models predict with regard to the invisible decays of the heavier $H$ and how this will impact possibilities for detecting the $H$ in upcoming LHC runs. For $m_{S} \lesssim 55 \mathrm{GeV}$, the $\mathrm{BR}(h \rightarrow S S) \leq 0.1$ constraint required by a good $h$ fit to the $125.5 \mathrm{GeV}$ data implies that $\lambda_{h}$ is small and this indirectly impacts $\operatorname{BR}(H \rightarrow S S)$. Before imposing the LUX limits, we find that $B R(H \rightarrow S S)$ can have a number of semi-discrete values below 1 , the discreteness being associated with particular $2 \mathrm{HDM} 68 \%$ C.L. points, but for the bulk of $m_{S} \lesssim 55 \mathrm{GeV}$ points one has $\mathrm{BR}(H \rightarrow S S) \gtrsim 0.9$. Of course, we have seen above that once the LUX and SuperCDMS limits are imposed all the low- $m_{S}$ points are eliminated in the Type I case, whereas in the Type II case a handful of points survive in the $m_{S} \leq 6 \mathrm{GeV}$ region. Once $m_{S} \gtrsim 55 \mathrm{GeV}$, 


\begin{tabular}{|c|c|c|c|c|c|c|}
\hline $\tan \beta$ & $\sin \alpha$ & $m_{H}$ & $m_{A}$ & $m_{H^{ \pm}}$ & $m_{12}^{2}$ & $\left(m_{S}[\mathrm{GeV}], \log \Theta_{\mathrm{Xe}}\left(f_{n}, f_{p}\right) \sigma_{S-p}\left[\mathrm{~cm}^{2}\right]\right)$ \\
\hline 2.092 & -0.41 & 138 & 451 & 399 & -12642 & $(3.44,-39.65) ;(3.56,-39.69) ;(3.95,-39.85)$ \\
\hline 3.121 & -0.282 & 187 & 546 & 571 & 8943 & $(4.82,-40.50) ;(5.48,-40.83)$ \\
\hline 2.192 & -0.394 & 209 & 488 & 503 & 7518 & $(5.40,-40.93)$ \\
\hline 1.728 & -0.476 & 177 & 318 & 389 & 9382 & $(5.16,-40.97)$ \\
\hline 1.789 & -0.461 & 198 & 420 & 430 & -6594 & $(4.44,-40.43) ;(5.15,-40.96)$ \\
\hline 1.488 & -0.528 & 157 & 553 & 576 & -10094 & $(4.61,-40.83)$ \\
\hline 2.375 & -0.363 & 259 & 260 & 339 & 15899 & $(5.83,-41.05)$ \\
\hline
\end{tabular}

Table 4. Summary of the properties of the 2HDM Type II points in figure 16 which make it possible to realize $m_{S}<50 \mathrm{GeV}$, after imposing the full set of preLUX constraints together with the LUX and SuperCDMS bounds. All masses are given in $\mathrm{GeV}$ units.

$\operatorname{BR}(h \rightarrow S S)$ is automatically small or zero and constraints on $\lambda_{h}$ in the singlet sector scan are greatly relaxed. As a result, $\mathrm{BR}(H \rightarrow S S)$ can take on most any value for $m_{S} \lesssim 200 \mathrm{GeV}$, declining to small values once $m_{S} \gtrsim 500 \mathrm{GeV}$.

As regards $H$ detection, we first note that since the $H V V$ couplings are small (since the $h V V$ coupling must be large for a good Higgs fit) the $Z+i n v$ final state LHC data do not currently constrain $\mathrm{BR}(H \rightarrow S S)$, and in future runs very high integrated luminosity would be needed to have any hope of seeing a signal in this channel. Further, if $H \rightarrow S S$ decays are dominant this would reduce the strength of the $H$ signals in other production/decay modes, such as $g g \rightarrow H \rightarrow \tau \tau$, and thus decrease the prospects for $H$ discovery as outlined in [32]. In such instances, experimental sensitivity to the $H$ may have to rely on $g g \rightarrow H$ production with a jet or photon tag of the invisible $H \rightarrow S S$ final state.

We now turn to an expanded discussion of the summary given above in which we split the scalar mass $m_{S}$ into three regions, depending on the status of the exotic decay $h \rightarrow S S$ :

- low mass region $(1-55 \mathrm{GeV})$ where the decay is open and could be substantial without $\lambda_{h}$ being very small;

- resonance region $(55-70 \mathrm{GeV})$ where $m_{S}$ is not far from the $h$ pole location. For $m_{S}<m_{h} / 2$, one finds that, after imposing $\mathrm{P}+\mathrm{S}+\mathrm{U}+\mathrm{EW}, \lambda_{h}$ is sufficiently limited that $\mathrm{BR}(h \rightarrow S S) \leq 0.1$. In fact, in this region, the strongest constraint on $\lambda_{h}$ comes from the need to avoid too much annihilation.

- high mass region $(70-1000 \mathrm{GeV})$ where the decay is absolutely closed.

Note that we adopt different scan strategies in these regions of $m_{S}$ so as to achieve a maximum density around the most interesting points that pass all theoretical and experimental constraints. The scans are also preformed in a different way for Type I and Type II models. 

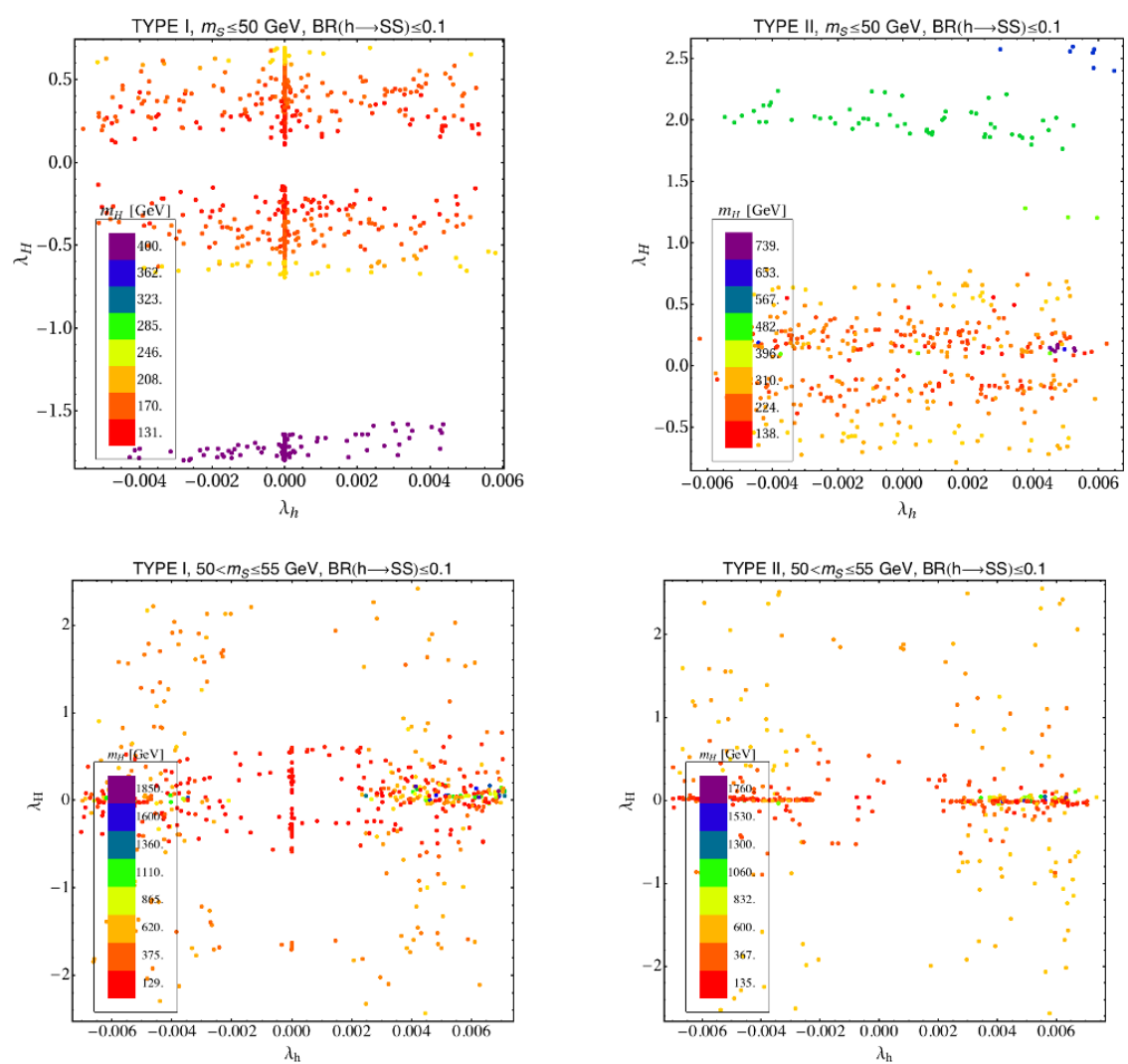

Figure 17. Couplings $\lambda_{h}, \lambda_{H}$ allowed by the full set of preLUX constraints for the $m_{S} \leq 55 \mathrm{GeV}$ mass region. Points are temperatured according to $m_{H}$, with red points corresponding to the lowest $H$ mass for which a solution was found. Upper figures are for $m_{S} \leq 50 \mathrm{GeV}$ while lower figures are for $50<m_{S} \leq 55 \mathrm{GeV}$.

\subsubsection{Low mass region}

As we have already noted, in the low mass region, the exotic decay $h \rightarrow S S$ could have a large branching ratio. In the case where a singlet scalar is added to the pure SM, one finds that the corresponding coupling of dark matter to the Higgs necessary to avoid overabundance of the relic $S$ is so large that $\mathrm{BR}\left(h_{\mathrm{SM}} \rightarrow S S\right) \gtrsim 0.9[35,40]$, thereby making a good fit of the $h_{\mathrm{SM}}$ to the LHC Higgs data impossible. In the 2HDMS, one can keep $\mathrm{BR}(h \rightarrow S S)$ small enough $(\leq 0.1)$ to avoid destroying the fit of the $h$ to the $125.5 \mathrm{GeV}$ Higgs data if $\lambda_{h} \ll 1$. Nonetheless, correct $\Omega h^{2}$ can be achieved because in the 2 HDMS the annihilation of DM is mediated not only by $h$ but also by $H$ (see figure 6 ). Therefore, the desired large cross section for $S S$ annihilation can be achieved if $\lambda_{H}$ is sufficiently large when $\lambda_{h}$ is small. This trend was already apparent in figure 9 . Here, we zero in on the $m_{h} \leq 55 \mathrm{GeV}$ region in figure 17, where we have employed a special scan strategy designed to cover a large range of $f_{n} / f_{p}$ and small $\lambda_{h}$. In the upper plots in figure 17, we require $m_{S} \leq 50 \mathrm{GeV}$ while the lower plots are for $50<m_{S} \leq 55 \mathrm{GeV}$. In the latter case, we observe a hole in the vicinity of small $\lambda_{h}$ and $\lambda_{H}$ which expands to a gap in the former case due to the fact that points with $m_{S} \leq 50 \mathrm{GeV}$ are sufficiently far from the resonance region that $H$ exchange, i.e. $\lambda_{H} \neq 0$, is needed for correct $\Omega h^{2}$. In contrast, for points with 
$50<m_{S} \leq 55 \mathrm{GeV}$, for $\lambda_{h} \neq 0$ the $h$ alone can provide enough annihilation for correct $\Omega h^{2}$ even if $\lambda_{H}=0$.

As expected, the temperature plots show that, generally speaking, the larger $m_{H}$ is the larger $\lambda_{H}$ must be for correct relic density (the $S S$ annihilation amplitude containing the ratio $\left.\lambda_{H} / m_{H}^{2}\right)$. However, there is an exception in the case of the Type II model; at large $\tan \beta(\gtrsim 25)$ one can have sufficient annihilation even if $\lambda_{H} / m_{H}^{2}$ is not large since the $H b \bar{b}$ coupling is highly enhanced, $C_{D}^{H} \propto \tan \beta$, see eq. (4.2). We observe a smattering of such points in the (upper) $m_{S} \leq 50 \mathrm{GeV}$ Type II plot. For these points, the $S S \rightarrow b \bar{b}$ annihilation cross section is large enough to produce relic abundance within the experimental limit even though $\left|\lambda_{H}\right|<0.2$ and $m_{H}>500 \mathrm{GeV}$.

We end this subsection with the plots of figure 18 showing the regions of the $2 \mathrm{HDM}$ parameter space with $m_{S} \leq 55 \mathrm{GeV}$ that remain after imposing the full set of preLUX constraints. The allowed regions are displayed in the $(\tan \beta, \sin \alpha),\left(m_{H}, m_{A}\right)$ and $\left(m_{H^{ \pm}}, m_{A}\right)$ planes. Different colors are used to distinguish those points with $m_{S} \leq 50$ from those with $50<m_{S} \leq 55 \mathrm{GeV}$. Also shown are those points that in addition satisfy the LUX limit.

\subsubsection{Resonance region}

In this subsection we focus on the $h$ resonance region, $55 \mathrm{GeV}<m_{S} \leq 70 \mathrm{GeV}$, which is defined such that the $h$ is near the pole of $S S$ annihilation, $m_{S} \approx m_{h} / 2$. In this region, the annihilation of $S S$ into SM particles is mainly mediated through exchanging an $s$-channel $h$ (unless the $H$ is not much heavier than the $h, m_{H} \approx m_{h}$ ). For a given magnitude of $\lambda_{h}$ (and $\lambda_{H}$ when $m_{H}$ is close to $m_{h}$ ), the annihilation cross section is greatly enhanced in the resonance region, as seen in Fig 12 for Type I and figure 16 for Type II, respectively. In order to compensate for the resonance enhancement, $\lambda_{h}$ and/or $\lambda_{H}$ in the resonance region must be small in order to reproduce the observed DM abundance, as shown in figure $19{ }^{8} \mathrm{In}$ the upper plots of this figure, we have imposed the full set of preLUX constraints including $B R(h \rightarrow S S) \leq 0.1$. In the lower plots, we have required that the LUX limits also be obeyed. We observe that this latter requirement reduces further the magnitudes of $\lambda_{h}$ and $\lambda_{H}$.

We also note that in this resonance region $B R(H \rightarrow S S)$ is typically large, between $\sim 0.1$ and $\sim 0.9$. If we were to repeat the plots of figure 18 for this case, we would find little change in the regions allowed, just an increase in point density. Indeed, very few of the starting $2 \mathrm{HDM}$ red points of figure 1 are eliminated by the preLUX constraints, implying that the regions shown are nearly identical to those for the original $2 \mathrm{HDM}$ points sampled. The reason for this is that once we are in the resonance region correct relic density can almost always be obtained by judiciously choosing $\lambda_{h}$ and $\lambda_{H}$.

\subsubsection{High mass region}

The high mass region is defined as $70 \leq m_{S} \leq 1000 \mathrm{GeV}$. In our study, the parameters $\kappa_{1}$ and $\kappa_{2}$ in the extra singlet sector are both scanned over in the range $\left(10^{-2}, 4 \pi\right)$ with

\footnotetext{
${ }^{8}$ In this mass range we scan over $\lambda_{h}$ and $\lambda_{H}$ from $\left(10^{-4}, 10^{-1}\right)$ and $\left(10^{-4}, 4 \pi\right)$, respectively, with logarithmic density.
} 

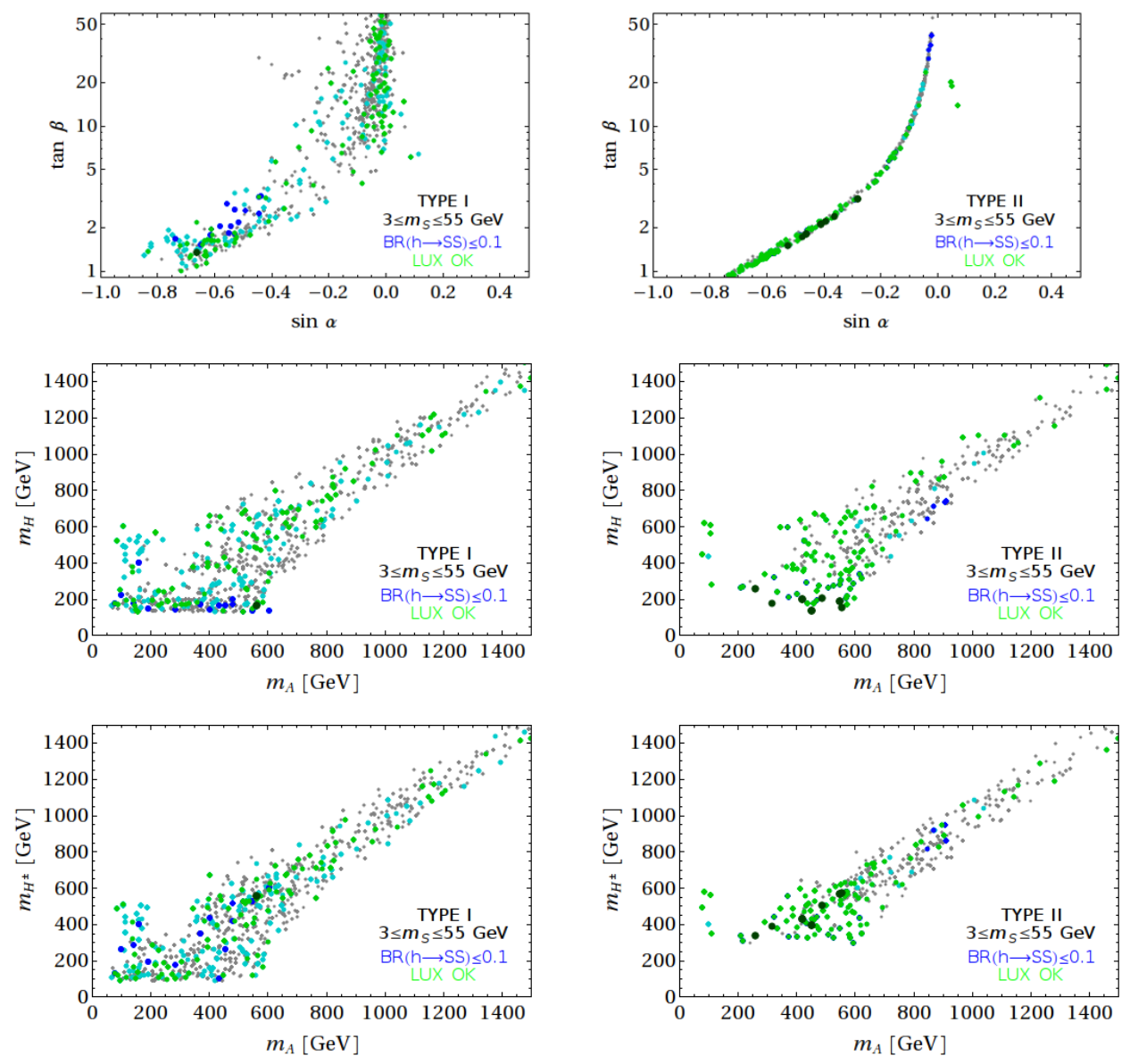

Figure 18. We show how the full set of preLUX constraints on the singlet sector affects the $2 \mathrm{HDM}$ parameter space that we used for the singlet sector scans. We have required $m_{S} \leq 55 \mathrm{GeV}$. Gray points satisfy all preLUX constraints aside from $\mathrm{BR}(h \rightarrow S S) \leq 0.1$. Cyan and blue points satisfy in addition $\operatorname{BR}(h \rightarrow S S) \leq 0.1$, i.e. the full set of preLUX constraints. Cyan points have $50<m_{S} \leq 55 \mathrm{GeV}$ while blue points have $m_{S} \leq 50 \mathrm{GeV}$. The green and dark green points satisfy the LUX bound as well as the full set of preLUX constraints, with dark green showing the $m_{S} \leq 50 \mathrm{GeV}$ points.

logarithmic density. Points surviving the full set of preLUX constraints are shown in the $\left(\lambda_{h}, \lambda_{H}\right)$ plane in the upper plots of figure 20. The lower plots show the points that also survive the LUX bound. There, one can observe that for high $m_{S}$ there is an ample parameter space surviving the preLUX constraints together with the LUX bound on the spin-independent cross section of DM direct detection.

\section{Conclusions}

We have analyzed the 2HDMS models obtained by extending the Type I and Type II twoHiggs-doublet models to include a scalar gauge-singlet dark matter candidate, denoted $S$ with mass $m_{S}$. We have discussed various theoretical and experimental constraints on the $2 \mathrm{HDMS}$ and how these constrain the additional (beyond the 2HDM) three parameters of 

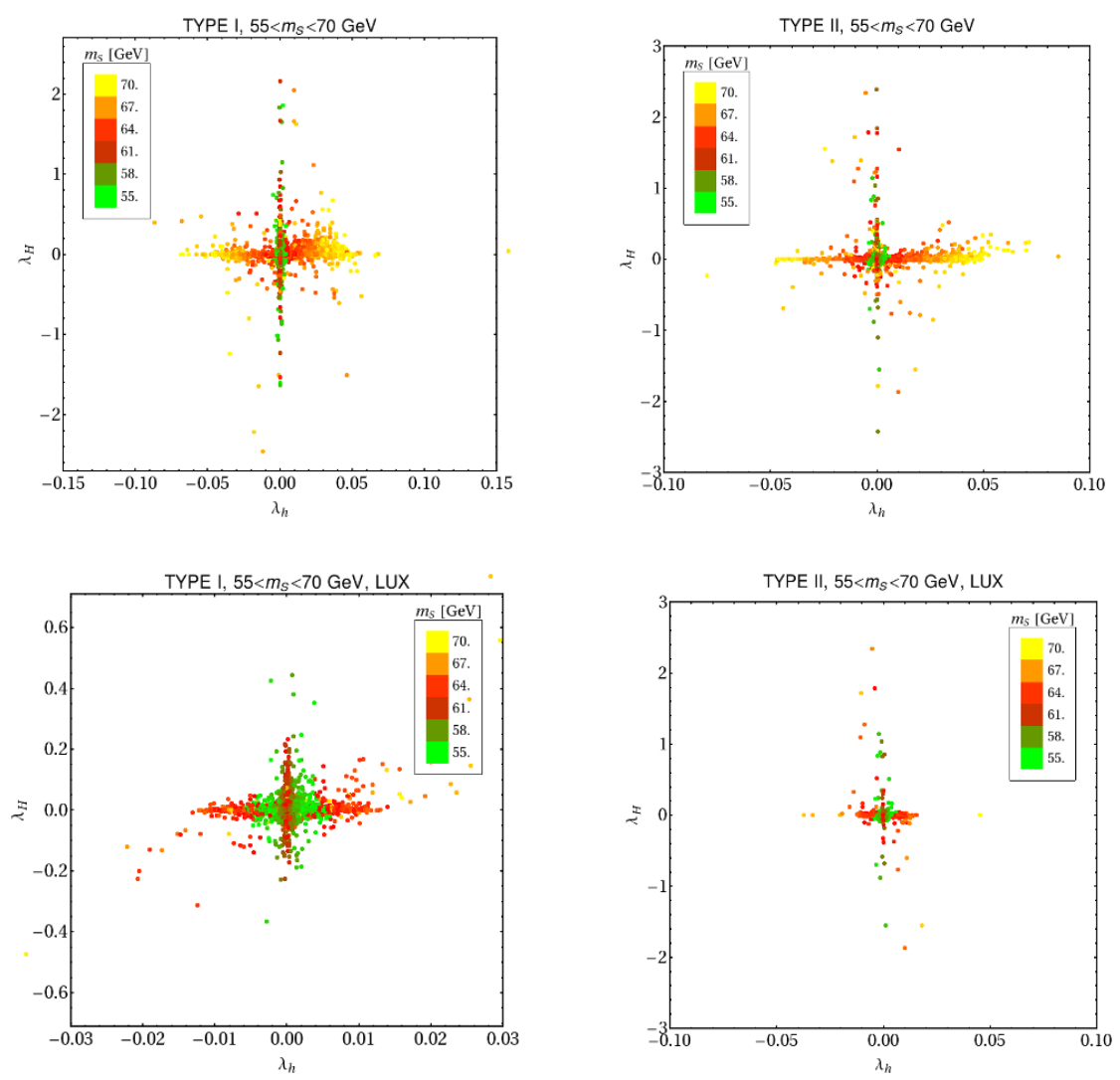

Figure 19. In the upper plots we show the couplings ouplings $\lambda_{h}, \lambda_{H}$ allowed by the full set of preLUX constraints when $m_{S}$ is in the resonance mass region, $55 \mathrm{GeV}<m_{S} \leq 70 \mathrm{GeV}$. In the lower plots, we show the points of the upper plots that are also consistent with the LUX limit on DM scattering.

the 2HDMS, $m_{S}$ and the trilinear $h S S$ and HSS couplings. We begin with the 2HDM fits of [32] for the case where it is the lighter $h$ that is identified with the $\sim 125.5 \mathrm{GeV}$ state, in particular employing the $2 \mathrm{HDM}$ parameter space points for which the combined LHC/Tevatron signal strengths are fit within the $68 \%$ C.L. We then study the constraints on the singlet parameter space based on cosmological data, most particularly the observed $\Omega h^{2}$ and the LUX and SuperCDMS limits on DM-nucleon scattering. If $m_{S}>55 \mathrm{GeV}$, 2HDMS parameter choices for which the 2 HDMS is completely consistent with all the above data are plentiful in both the Type I and Type II models. For $m_{S} \leq 55 \mathrm{GeV}$, requiring $\mathrm{BR}(h \rightarrow S S) \leq 0.1$ in order to avoid destroying the fit of the $h$ to the LHC data makes it impossible (almost impossible) in the Type I (Type II) model to find parameter points that give correct $\Omega h^{2}$ and satisfy both the LUX and SuperCDMS limits. Nonetheless, it is interesting to note that if we do not impose the LUX and SuperCDMS limits, for both model types $m_{S}<50 \mathrm{GeV}$-points with $\mathrm{BR}(h \rightarrow S S) \leq 0.1$ and correct $\Omega h^{2}$ fall within one or more of the CDMS II, CRESST-II or CoGeNT signal regions.

An important issue in the $2 \mathrm{HDMS}$ context is whether or not there is a possibility of isospin violation, $f_{n} / f_{p} \neq 1$. In the case of the 2 HDMS Type I model, $f_{n} / f_{p} \simeq 1$ is inevitable. This, implies that despite the fact that all points with correct $\Omega h^{2}$ and $\operatorname{BR}(h \rightarrow$ 

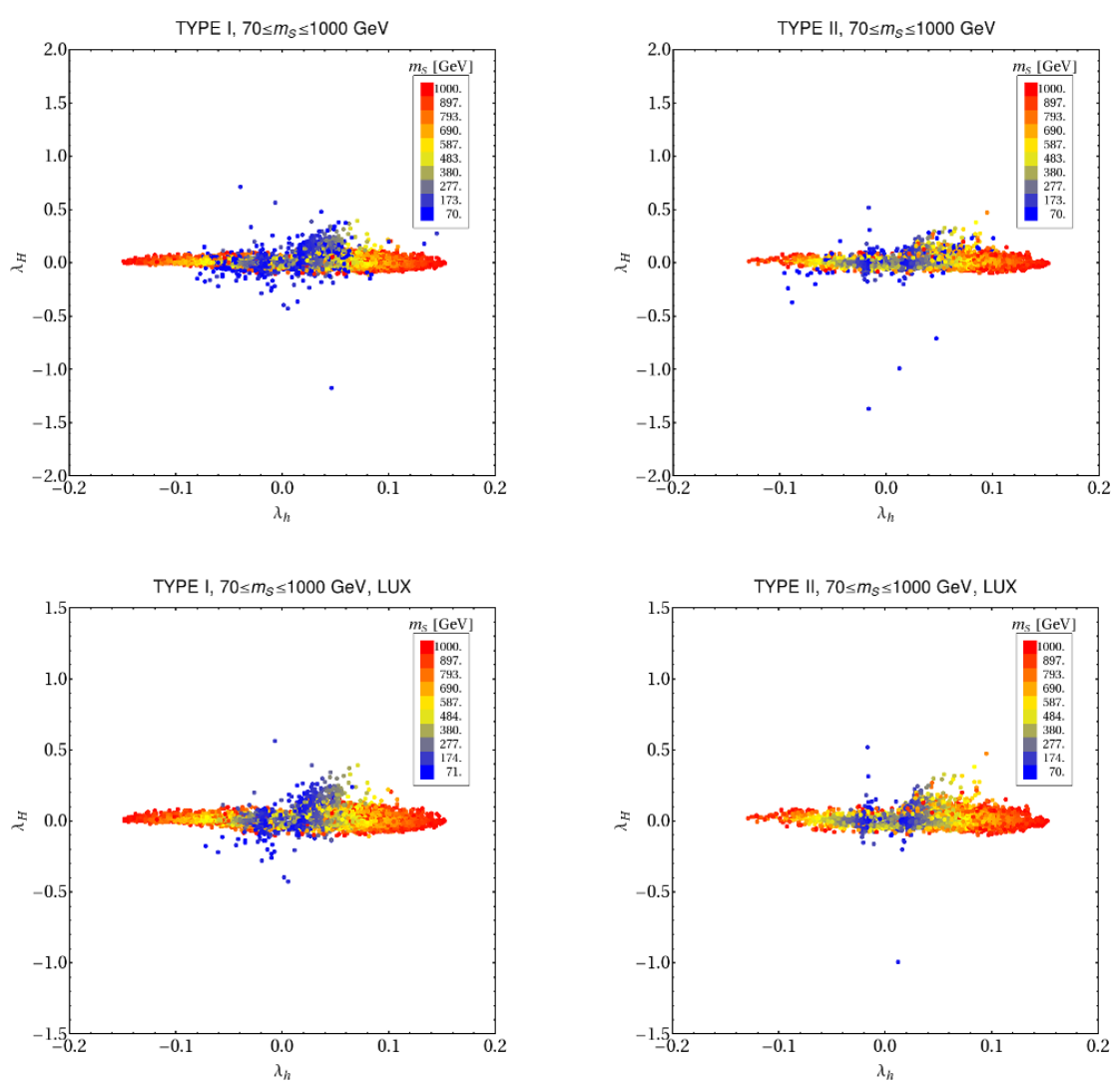

Figure 20. Couplings $\lambda_{h}, \lambda_{H}$ allowed by the full set of preLUX constraints for $m_{S}$ within the high mass region. The LUX limit is imposed on the points shown in the lower plots.

$S S) \leq 0.1$ have $\sigma_{S-p}$ values falling within one or more of the CDMS II, CRESST-II or CoGeNT signal regions, they are simply inconsistent with the LUX and SuperCDMS limits.

In the case of the 2HDMS Type II model, a significant isospin violation in DM-nucleon scattering is possible, even reaching the value of $f_{n} / f_{p} \sim-0.7$ that would allow consistency of the LUX limit with the CDMS II signal region. However, at the low $m_{S}$ values corresponding to the signal region, we find that points with $f_{n} / f_{p} \sim-0.7$ either have an $h S S$ coupling that is too large for $\operatorname{BR}(h \rightarrow S S) \leq 0.1$ or too small to give sufficient annihilation to achieve correct $\Omega h^{2}$. (At low $m_{S}$, the $H$ exchange contribution to $S S$ annihilation is not sufficient, given upper bounds on the $H S S$ coupling coming from perturbativity and unitarity.) Therefore, even though isospin violation might be present, the level of $f_{n} / f_{p} \sim-0.7$ cannot be made consistent with all phenomenological requirements. The SuperCDMS limit further constrains the picture. For the $f_{n} / f_{p}$ values predicted by the 2HDMS once correct $\Omega h^{2}$ and $\mathrm{BR}(h \rightarrow S S) \leq 0.1$ are imposed, the isospin violation is only a small effect in comparing the Germanium target SuperCDMS limit to the Silicon target CDMS II result. In the end, one does find a few $m_{S} \sim 5.5-6.2 \mathrm{GeV}$-points that lie below both the SuperCDMS and LUX limits and, interestingly, also fall within (but are outside) the $99 \%$ C.L. (95\% C.L.) CDMS II signal region. As typical for $m_{S} \leq 50 \mathrm{GeV}$, these points are such that $\mathrm{BR}(H \rightarrow S S)$ is large, implying that jet- and/or photon-tagging will be needed for $H$ detection. 


\section{A Vacuum stability}

We are considering 2HDMS scalar potential with $\lambda_{6}=\lambda_{7}=0, \lambda_{5} \in R$ and $\kappa_{3}=0$ :

$$
\begin{aligned}
V\left(H_{1}, H_{2}, S\right)= & \frac{\lambda_{1}}{2}\left(H_{1}^{\dagger} H_{1}\right)^{2}+\frac{\lambda_{2}}{2}\left(H_{2}^{\dagger} H_{2}\right)^{2}+\lambda_{3}\left(H_{1}^{\dagger} H_{1}\right)\left(H_{2}^{\dagger} H_{2}\right)+\lambda_{4}\left|H_{1}^{\dagger} H_{2}\right|^{2} \\
& +\left[\frac{\lambda_{5}}{2}\left(H_{1}^{\dagger} H_{2}\right)^{2}+\text { h.c. }\right]+\frac{1}{4 !} \lambda_{S} S^{4}+\kappa_{1} S^{2}\left(H_{1}^{\dagger} H_{1}\right)+\kappa_{2} S^{2}\left(H_{2}^{\dagger} H_{2}\right)
\end{aligned}
$$

We will use the following reparametrization of the potential [74, 75]:

$$
\begin{aligned}
\left|H_{1}\right| & =r \cos \theta \sin \phi, \quad \quad\left|H_{2}\right|=r \sin \theta \sin \phi, \\
H_{1}^{\dagger} H_{2} & =\left|H_{1}\right|\left|H_{2}\right| \rho e^{i \gamma}, \quad S=r \cos \phi
\end{aligned}
$$

Because $\frac{H_{1}^{\dagger}}{\left|H_{1}\right|} \cdot \frac{H_{2}}{\left|H_{2}\right|}$ is a product of unit spinors, it is a complex number $\alpha+i \beta$ such that $|\alpha+i \beta|<=1$. I can rewrite it in polar coordinates as $\alpha+i \beta=\rho e^{i \gamma}$ with $\rho \in(0,1)$. Rewriting the potential in terms of $r, \theta, \phi, \rho, \gamma$ we get

$$
\begin{aligned}
& V / r^{4}=( \frac{\lambda_{1}}{2} \cos ^{4} \theta+\frac{\lambda_{2}}{2} \sin ^{4} \theta+\lambda_{3} \cos ^{2} \theta \sin ^{2} \theta \\
&\left.+\lambda_{4} \rho^{2} \sin ^{2} \theta \cos ^{2} \theta+\lambda_{5} \rho^{2} \sin ^{2} \theta \cos ^{2} \theta \cos (2 \gamma)\right) \sin ^{4} \phi \\
&+\frac{1}{4 !} \lambda_{S} \cos ^{4} \phi+\kappa_{1} \cos ^{\theta} \sin ^{\phi} \cos ^{2} \phi+\kappa_{2} \sin ^{2} \theta \cos ^{2} \phi \sin ^{2} \phi
\end{aligned}
$$

where $V=V\left(\cos ^{2} \theta, \sin ^{2} \phi, \cos (2 \gamma), \rho\right)$ and this parameters change in the following ranges:

$$
\begin{array}{ll}
x=\cos ^{2} \theta \in(0,1), & y=\sin ^{2} \phi \in(0,1), \\
z=\cos (2 \gamma) \in(-1,1), & \rho \in(0,1)
\end{array}
$$

and we can rewrite the potential in the final form:

$$
\begin{aligned}
V / r^{4}= & \left(\frac{\lambda_{1}}{2} x^{2}+\frac{\lambda_{2}}{2}(1-x)^{2}+\lambda_{3} x(1-x)+\lambda_{4} \rho^{2} x(1-x)+\lambda_{5} \rho^{2} x(1-x) z\right) y^{2} \\
& +\frac{1}{4 !} \lambda_{S}(1-y)^{2}+\left(\kappa_{1} x+\kappa_{2}(1-x)\right) y(1-y)
\end{aligned}
$$

To assure stability potential has to be bounded from below, which means that in the limit of infinite fields $V$ has to approach $+\infty$. Therefore the necessary and sufficient condition for tree level stability of our theory is a positive minimum of $V / r^{4}$ in (A.5). Let's use a following lemma to find constraints on potential parameters for which the minimum is indeed positive:

\section{Lemma 1.}

$$
f(\xi)=a \xi^{2}+b(1-\xi)^{2}+c \xi(1-\xi)>0, \quad \xi \in(0,1) \Longleftrightarrow a>0, b>0, c>-\sqrt{4 a b}
$$


This can be easily shown to be true using basic properties of a quadratic function. Lemma 1 for $\xi=y$ leads to the following set of constraints:

$$
\begin{aligned}
& A=\left(\frac{\lambda_{1}}{2} x^{2}+\frac{\lambda_{2}}{2}(1-x)^{2}+\lambda_{3} x(1-x)+\lambda_{4} \rho^{2} x(1-x)+\lambda_{5} \rho^{2} x(1-x) z\right)>0 \\
& B=\frac{1}{4 !} \lambda_{S}>0 \\
& C=\left(\kappa_{1} x+\kappa_{2}(1-x)\right)>-\sqrt{4 A B}
\end{aligned}
$$

where $A>0$ is the regular $2 \mathrm{HDM}$ constraint and $B>0$ leads to $\lambda_{S}>0$. We can rewrite $C>-\sqrt{4 A B}$ in a way to use Lemma $I$ again.

$$
\begin{aligned}
0<\left(\frac{1}{12} \lambda_{S} \lambda_{1}\right. & \left.-\kappa_{1}^{2}\right) x^{2}+\left(\frac{1}{12} \lambda_{S} \lambda_{2}-\kappa_{2}^{2}\right)(1-x)^{2} \\
& +\left(-2 \kappa_{1} \kappa_{2}+\frac{1}{6} \lambda_{S}\left(\lambda_{3}+\lambda_{4} \rho^{2}+\lambda_{5} \rho^{2} z\right)\right) x(1-x)
\end{aligned}
$$

This form leads to a following set of inequalities:

$$
\begin{aligned}
A^{\prime} & =\left(\frac{1}{12} \lambda_{S} \lambda_{1}-\kappa_{1}^{2}\right)>0 \\
B^{\prime} & =\left(\frac{1}{12} \lambda_{S} \lambda_{2}-\kappa_{2}^{2}\right)>0 \\
C^{\prime} & =\left(\frac{1}{6} \lambda_{S}\left(\lambda_{3}+\lambda_{4} \rho^{2}+\lambda_{5} \rho^{2} z\right)\right)>-\sqrt{4 A^{\prime} B^{\prime}}
\end{aligned}
$$

The full set of stability constraints on 2HDMS potential is the following:

- $\lambda_{1}, \lambda_{2}, \lambda_{S}>0$

- $\lambda_{3}+\lambda_{4}-\left|\lambda_{5}\right|>-\sqrt{\lambda_{1} \lambda_{2}}$

- $\lambda_{3}>-\sqrt{\lambda_{1} \lambda_{2}}$

- $\kappa_{1}>-\sqrt{\frac{1}{12} \lambda_{S} \lambda_{1}}$

- $\kappa_{2}>-\sqrt{\frac{1}{12} \lambda_{S} \lambda_{2}}$

- if $\kappa_{1}$ or $\kappa_{2}<0$ then we have to satisfy also:

$$
\begin{aligned}
& { }^{*}-2 \kappa_{1} \kappa_{2}+\frac{1}{6} \lambda_{S} \lambda_{3}>-\sqrt{4\left(\frac{1}{12} \lambda_{S} \lambda_{1}-\kappa_{1}^{2}\right)\left(\frac{1}{12} \lambda_{S} \lambda_{2}-\kappa_{2}^{2}\right)} \\
& *-2 \kappa_{1} \kappa_{2}+\frac{1}{6} \lambda_{S}\left(\lambda_{3}+\lambda_{4}-\left|\lambda_{5}\right|\right)>-\sqrt{4\left(\frac{1}{12} \lambda_{S} \lambda_{1}-\kappa_{1}^{2}\right)\left(\frac{1}{12} \lambda_{S} \lambda_{2}-\kappa_{2}^{2}\right)}
\end{aligned}
$$




\section{B Tree-level perturbative unitarity}

A natural way for derivation of the tree-level unitarity constraints is to construct the full multi-state scattering matrix for all the physical scalar-scalar states (including possible states associated with longitudinal vector bosons) in the tree approximation at high enough energy (where the scattering is dominantly mediated by the direct quartic interactions), requiring its largest eigenvalue to be less than the upper limit, i.e. 16 $\pi$. This way involves a large dimensional matrix for which determining the eigenvalues is usually impractical. Alternatively, this limitations for eigenvalues of scattering matrix can be obtained in any basis related to the physical basis by a unitarity transformation. It was shown in [7678 ] that the derivation for the $2 \mathrm{HDM}$ is considerably simple in the basis of non-physical electroweak eigenstates although it still needs explicit work with components of Higgs doublets. An even simpler approach dealing with the initial doublets $H_{1}$ and $H_{2}$ was developed in [79]. In this paper we will adopt the technique introduced in [77] and extend the derivation to the $2 \mathrm{HDM}$ plus a singlet model, taking into account an additional singlet in constructing the full scattering matrix.

Following the previous studies [76-78], we start with the most general 2HDMS potential in the HHG parametrization that is subject to

$$
\begin{aligned}
V\left(\Phi_{1}, \Phi_{2}\right)= & \Lambda_{1}\left(\left|H_{1}\right|^{2}-\frac{1}{2} v_{1}^{2}\right)^{2}+\Lambda_{2}\left(\left|H_{2}\right|^{2}-\frac{1}{2} v_{2}^{2}\right)^{2} \\
& +\Lambda_{3}\left[\left(\left|H_{1}\right|^{2}-\frac{1}{2} v_{1}^{2}\right)+\left(\left|H_{2}\right|^{2}-\frac{1}{2} v_{2}^{2}\right)\right]^{2} \\
& +\Lambda_{4}\left(\left|H_{1}\right|^{2}\left|H_{2}\right|^{2}-\left|H_{1}^{+} H_{2}\right|^{2}\right)+\Lambda_{5}\left[\Re\left(H_{1}^{+} H_{2}\right)-\frac{1}{2} v_{1} v_{2}\right]^{2}+\Lambda_{6}\left[\Im\left(H_{1}^{+} H_{2}\right)\right]^{2} \\
& +\frac{1}{2} m_{0}^{2} S^{2}+\frac{1}{4 !} \lambda_{S} S^{4}+\kappa_{1} S^{2}\left(H_{1}^{\dagger} H_{1}\right)+\kappa_{2} S^{2}\left(H_{2}^{\dagger} H_{2}\right)
\end{aligned}
$$

where the $\Lambda_{i}$ are real parameters.

$$
H_{1}=\left(\begin{array}{c}
w_{1}^{+} \\
\frac{1}{\sqrt{2}}\left(v_{1}+h_{1}+i z_{1}\right)
\end{array}\right), \quad H_{2}=\left(\begin{array}{c}
w_{2}^{+} \\
\frac{1}{\sqrt{2}}\left(v_{2}+h_{2}+i z_{2}\right)
\end{array}\right)
$$

In principle, one needs to examine the full scattering matrix, including all two-particle states made of the scalars (including the unphysical Goldstone bosons) $w_{i}^{ \pm}, h_{i}, z_{i}(i=$ $1,2)$ and $s$. Nonetheless, charge conservation and CP-invariance forbids some scattering processes, making it composed by four sub-matrices, as illustrated in [77, 78].

Without the help of the $s$ state originated from the singlet $S$ sector, one can construct the extra charged states $\left(w_{1}^{+} s, w_{2}^{+} s\right)$ which decouple with the other charged states $\left(w_{1}^{+} h_{1}, w_{1}^{+} h_{2}, w_{1}^{+} z_{1}, w_{1}^{+} z_{2}, w_{2}^{+} h_{1}, w_{2}^{+} h_{2}, w_{2}^{+} z_{1}, w_{2}^{+} z_{2}\right)$ existed in the 2HDM. Only the last two terms in eq. (B.1) handle the scattering matrix under this basis, which is given by

$$
\mathcal{M}=\left(\begin{array}{cc}
2 \kappa_{1} & 0 \\
0 & 2 \kappa_{2}
\end{array}\right)
$$

It is apparently diagonal and has eigenvalues $2 \kappa_{1}, 2 \kappa_{2}$. 
However, the neutral states under the basis $\left(w_{1}^{+} w_{1}^{-}, w_{2}^{+} w_{2}^{-}, \frac{z_{1} z_{1}}{\sqrt{2}}, \frac{h_{1} h_{1}}{\sqrt{2}}, \frac{z_{2} z_{2}}{\sqrt{2}}, \frac{h_{2} h_{2}}{\sqrt{2}}, \frac{s s}{\sqrt{2}}\right)$ is more involved. We have to write out the full scattering matrix,

$$
\mathcal{M}=\left(\begin{array}{ccccccc}
4 \Lambda_{13}^{+} & 2 \Lambda_{3}+\frac{\Lambda_{56}^{+}}{2} & \sqrt{2} \Lambda_{13}^{+} & \sqrt{2} \Lambda_{13}^{+} & \sqrt{2} \tilde{\Lambda}_{34}^{+} & \sqrt{2} \tilde{\Lambda}_{34}^{+} & \sqrt{2} \kappa_{1} \\
2 \Lambda_{3}+\frac{\Lambda_{56}^{+}}{2} & 4 \Lambda_{23}^{+} & \sqrt{2} \tilde{\Lambda}_{34}^{+} & \sqrt{2} \tilde{\Lambda}_{34}^{+} & \sqrt{2} \Lambda_{23}^{+} & \sqrt{2} \Lambda_{23}^{+} & \sqrt{2} \kappa_{2} \\
\sqrt{2} \Lambda_{13}^{+} & \sqrt{2} \tilde{\Lambda}_{34}^{+} & 3 \Lambda_{13}^{+} & \Lambda_{13}^{+} & \tilde{\Lambda}_{35}^{+} & \tilde{\Lambda}_{36}^{+} & \kappa_{1} \\
\sqrt{2} \Lambda_{13}^{+} & \sqrt{2} \tilde{\Lambda}_{34}^{+} & \Lambda_{13}^{+} & 3 \Lambda_{13}^{+} & \tilde{\Lambda}_{36}^{+} & \tilde{\Lambda}_{35}^{+} & \kappa_{1} \\
\sqrt{2} \tilde{\Lambda}_{34}^{+} & \sqrt{2} \Lambda_{23} & \tilde{\Lambda}_{35}^{+} & \tilde{\Lambda}_{36}^{+} & 3 \Lambda_{23} & \Lambda_{23} & \kappa_{2} \\
\sqrt{2} \tilde{\Lambda}_{34}^{+} & \sqrt{2} \Lambda_{23} & \tilde{\Lambda}_{36}^{+} & \tilde{\Lambda}_{35}^{+} & \Lambda_{23} & 3 \Lambda_{23} & \kappa_{2} \\
\sqrt{2} \kappa_{1} & \sqrt{2} \kappa_{2} & \kappa_{1} & \kappa_{1} & \kappa_{2} & \kappa_{2} & \frac{1}{2} \lambda_{S}
\end{array}\right)
$$

where $\Lambda_{i j}^{+}=\Lambda_{i}+\Lambda_{j}$ and $\tilde{\Lambda}_{i j}^{+}=\Lambda_{i}+\frac{\Lambda_{j}}{2}$ with

$$
\begin{aligned}
& \Lambda_{1}=\frac{1}{2}\left[\lambda_{1}-\left(\lambda_{3}+\lambda_{4}+\lambda_{5}\right)+2 m_{12}^{2} /\left(v^{2} s_{\beta} c_{\beta}\right)\right], \\
& \Lambda_{2}=\frac{1}{2}\left[\lambda_{2}-\left(\lambda_{3}+\lambda_{4}+\lambda_{5}\right)+2 m_{12}^{2} /\left(v^{2} s_{\beta} c_{\beta}\right)\right], \\
& \Lambda_{3}=\frac{1}{2}\left[\left(\lambda_{3}+\lambda_{4}+\lambda_{5}\right)-2 m_{12}^{2} /\left(v^{2} s_{\beta} c_{\beta}\right)\right], \\
& \Lambda_{4}=2 m_{12}^{2} /\left(v^{2} s_{\beta} c_{\beta}\right)-\lambda_{4}-\lambda_{5}, \\
& \Lambda_{5}=2 m_{12}^{2} /\left(v^{2} s_{\beta} c_{\beta}\right), \\
& \Lambda_{6}=2 m_{12}^{2} /\left(v^{2} s_{\beta} c_{\beta}\right)-2 \lambda_{5} .
\end{aligned}
$$

The analytical form of eigenvalues are

$$
\begin{aligned}
& b_{ \pm}=\Lambda_{1}+\Lambda_{2}+2 \Lambda_{3} \pm \sqrt{\left(\Lambda_{1}-\Lambda_{2}\right)^{2}+\frac{1}{4}\left(2 \Lambda_{4}-\Lambda_{5}-\Lambda_{6}\right)^{2}} \\
& c_{ \pm}=\Lambda_{1}+\Lambda_{2}+2 \Lambda_{3} \pm \sqrt{\left(\Lambda_{1}-\Lambda_{2}\right)^{2}+\frac{1}{4}\left(\Lambda_{5}-\Lambda_{6}\right)^{2}}
\end{aligned}
$$

and the rest three ones $a_{1,2,3}$ comes from the cubic polynomial equation

$$
\begin{aligned}
& x^{3}-12\left(\tilde{\Lambda}_{321}^{+}+\frac{1}{24} \lambda_{S}\right) x^{2} \\
& +\left[36 \bar{\Lambda}_{123}+\left(2 \Lambda_{3}-\tilde{\Lambda}_{456}^{+}\right)\left(10 \Lambda_{3}+\tilde{\Lambda}_{456}^{+}\right)+6 \lambda_{S} \tilde{\Lambda}_{321}^{+}-4\left(\kappa_{1}^{2}+\kappa_{2}^{2}\right)\right] x \\
& \quad-18 \lambda_{S} \bar{\Lambda}_{123}-\frac{1}{2} \lambda_{S}\left(2 \Lambda_{3}-\tilde{\Lambda}_{456}^{+}\right)\left(10 \Lambda_{3}+\tilde{\Lambda}_{456}^{+}\right) \\
& \quad+24\left(\kappa_{1}^{2} \Lambda_{23}^{+}+\kappa_{2}^{2} \Lambda_{13}^{+}\right)-8 \kappa_{1} \kappa_{2}\left(4 \Lambda_{3}+\tilde{\Lambda}_{456}^{+}\right)=0
\end{aligned}
$$

where $\tilde{\Lambda}_{i j k}^{+}=\Lambda_{i}+\frac{\Lambda_{j}+\Lambda_{k}}{2}$ and $\bar{\Lambda}_{i j k}=\Lambda_{i} \Lambda_{j}+\Lambda_{i} \Lambda_{k}+\Lambda_{j} \Lambda_{k}$.

Another two scattering submatrices under the neutral states basis $\left(h_{1} s, z_{1} s\right)$ and $\left(h_{2} s, z_{2} s\right)$ are both diagonal and has eigenvalues $2 \kappa_{1}$ and $2 \kappa_{2}$, respectively. 
Finally, the remaining $S$-matrix for $2-2$ process is unchanged and was already studied in [78]. The eigenvalues which have significant impacts on preventing the unitarity bound are

$$
\begin{aligned}
& e_{1}=2 \Lambda_{3}-\Lambda_{4}-\frac{1}{2} \Lambda_{5}+\frac{5}{2} \Lambda_{6} \\
& f_{+}=2 \Lambda_{3}-\Lambda_{4}+\frac{5}{2} \Lambda_{5}-\frac{1}{2} \Lambda_{6} \\
& p_{1}=2\left(\Lambda_{3}+\Lambda_{4}\right)-\frac{1}{2} \Lambda_{5}-\frac{1}{2} \Lambda_{6}
\end{aligned}
$$

Putting all together, it is thus sufficient to taking the inequalities into account as follows

$$
\max \left\{\left|a_{1,2,3}\right|\right\},\left|f_{+}\right|,\left|e_{1}\right|,\left|p_{1}\right|, 2 \kappa_{1}, 2 \kappa_{2} \leq 8 \pi
$$

\section{Acknowledgments}

JFG and YJ are supported in part by US DOE grant DE-SC-000999. YJ is also supported by LHC-TI fellowship US NSF grant PHY-0969510. AD and BG are supported by the National Science Centre (Poland) under research project, decision no DEC-2011/01/B/ST2/00438 and by the Foundation for Polish Science International PhD Projects Programme co-financed by the EU European Regional Development Fund. JFG and YJ thank Warsaw University for support at various stage of this project. YJ also acknowledges partial support by the Mainz Dark Matter Workshop where the early plan of this project was presented. JFG and YJ thank Beranger Dumont and Sabine Kraml for their collaboration on the $2 \mathrm{HDM}$ data base employed as part of the present work.

Open Access. This article is distributed under the terms of the Creative Commons Attribution License (CC-BY 4.0), which permits any use, distribution and reproduction in any medium, provided the original author(s) and source are credited.

\section{References}

[1] LUX collaboration, D.S. Akerib et al., First results from the LUX dark matter experiment at the Sanford Underground Research Facility, Phys. Rev. Lett. 112 (2014) 091303 [arXiv: 1310.8214] [INSPIRE].

[2] SuperCDMS collaboration, R. Agnese et al., Search for low-mass WIMPs with SuperCDMS, Phys. Rev. Lett. 112 (2014) 241302 [arXiv:1402.7137] [INSPIRE].

[3] XENON100 collaboration, E. Aprile et al., Dark matter results from 225 live days of XENON100 data, Phys. Rev. Lett. 109 (2012) 181301 [arXiv:1207.5988] [InSPIRE].

[4] CDMS collaboration, R. Agnese et al., Silicon detector results from the first five-tower run of CDMS II, Phys. Rev. D 88 (2013) 031104 [arXiv: 1304.3706] [InSPIRE].

[5] CDMS collaboration, R. Agnese et al., Silicon detector dark matter results from the final exposure of CDMS II, Phys. Rev. Lett. 111 (2013) 251301 [arXiv:1304.4279] [INSPIRE].

[6] R. Bernabei et al., Final model independent result of DAMA/LIBRA-phase1, Eur. Phys. J. C 73 (2013) 2648 [arXiv:1308.5109] [INSPIRE]. 
[7] CoGeNT collaboration, C.E. Aalseth et al., Results from a search for light-mass dark matter with a p-type point contact germanium detector, Phys. Rev. Lett. 106 (2011) 131301 [arXiv: 1002.4703] [INSPIRE].

[8] C.E. Aalseth et al., Maximum likelihood signal extraction method applied to 3.4 years of CoGeNT data, arXiv:1401.6234 [INSPIRE].

[9] G. Angloher et al., Results from $730 \mathrm{~kg}$ days of the CRESST-II dark matter search, Eur. Phys. J. C 72 (2012) 1971 [arXiv:1109.0702] [InSPIRE].

[10] J.L. Feng, J. Kumar, D. Marfatia and D. Sanford, Isospin-violating dark matter, Phys. Lett. B 703 (2011) 124 [arXiv:1102.4331] [INSPIRE].

[11] M.T. Frandsen, F. Kahlhoefer, C. McCabe, S. Sarkar and K. Schmidt-Hoberg, The unbearable lightness of being: CDMS versus XENON, JCAP 07 (2013) 023 [arXiv: 1304.6066] [INSPIRE].

[12] J.L. Feng, J. Kumar and D. Sanford, Xenophobic dark matter, Phys. Rev. D 88 (2013) 015021 [arXiv: 1306.2315] [INSPIRE].

[13] E. Del Nobile, G.B. Gelmini, P. Gondolo and J.-H. Huh, Update on light WIMP limits: LUX, lite and light, JCAP 03 (2014) 014 [arXiv:1311.4247] [INSPIRE].

[14] V. Cirigliano, M.L. Graesser, G. Ovanesyan and I.M. Shoemaker, Shining LUX on isospin-violating dark matter beyond leading order, arXiv:1311.5886 [INSPIRE].

[15] N. Chen et al., Exothermic isospin-violating dark matter after SuperCDMS and CDEX, arXiv: 1404.6043 [INSPIRE].

[16] G.B. Gelmini, A. Georgescu and J.-H. Huh, Direct detection of light "Ge-phobic" exothermic dark matter, JCAP 07 (2014) 028 [arXiv: 1404.7484] [INSPIRE].

[17] C.-W. Chiang and K. Yagyu, Implications of Higgs boson search data on the two-Higgs doublet models with a softly broken $Z_{2}$ symmetry, JHEP 07 (2013) 160 [arXiv:1303.0168] [INSPIRE].

[18] B. Grinstein and P. Uttayarat, Carving out parameter space in type-II two Higgs doublets model, JHEP 06 (2013) 094 [Erratum ibid. 1309 (2013) 110] [arXiv:1304. 0028] [INSPIRE].

[19] B. Coleppa, F. Kling and S. Su, Constraining type II 2HDM in light of LHC Higgs searches, JHEP 01 (2014) 161 [arXiv:1305.0002] [INSPIRE].

[20] C.-Y. Chen, S. Dawson and M. Sher, Heavy Higgs searches and constraints on two Higgs doublet models, Phys. Rev. D 88 (2013) 015018 [arXiv:1305.1624] [INSPIRE].

[21] O. Eberhardt, U. Nierste and M. Wiebusch, Status of the two-Higgs-doublet model of type-II, JHEP 07 (2013) 118 [arXiv: 1305.1649] [InSPIRE].

[22] N. Craig, J. Galloway and S. Thomas, Searching for signs of the second Higgs doublet, arXiv:1305.2424 [INSPIRE].

[23] V. Barger, L.L. Everett, H.E. Logan and G. Shaughnessy, Scrutinizing the 125 GeV Higgs boson in two Higgs doublet models at the LHC, ILC and Muon Collider, Phys. Rev. D 88 (2013) 115003 [arXiv:1308.0052] [INSPIRE].

[24] S. Chang et al., Two Higgs doublet models for the LHC Higgs boson data at $\sqrt{s}=7$ and $8 \mathrm{TeV}$, JHEP 09 (2014) 101 [arXiv:1310.3374] [INSPIRE].

[25] K. Cheung, J.S. Lee and P.-Y. Tseng, Higgcision in the two-Higgs doublet models, JHEP 01 (2014) 085 [arXiv:1310.3937] [INSPIRE]. 
[26] A. Celis, V. Ilisie and A. Pich, Towards a general analysis of LHC data within two-Higgs-doublet models, JHEP 12 (2013) 095 [arXiv: 1310.7941] [INSPIRE].

[27] L. Wang and X.-F. Han, Status of the aligned two-Higgs-doublet model confronted with the Higgs data, JHEP 04 (2014) 128 [arXiv: 1312.4759] [INSPIRE].

[28] J. Baglio, O. Eberhardt, U. Nierste and M. Wiebusch, Benchmarks for Higgs pair production and heavy Higgs searches in the two-Higgs-doublet model of type II, Phys. Rev. D 90 (2014) 015008 [arXiv: 1403.1264 ] [INSPIRE].

[29] P.M. Ferreira, J.F. Gunion, H.E. Haber and R. Santos, Probing wrong-sign Yukawa couplings at the LHC and a future linear collider, Phys. Rev. D 89 (2014) 115003 [arXiv:1403.4736] [INSPIRE].

[30] S. Kanemura, H. Yokoya and Y.-J. Zheng, Complementarity in direct searches for additional Higgs bosons at the LHC and the International Linear Collider, Nucl. Phys. B 886 (2014) 524 [arXiv: 1404.5835] [INSPIRE].

[31] L. Wang and X.-F. Han, Study of the heavy CP-even Higgs with mass $125 \mathrm{GeV}$ in two-Higgs-doublet models at the LHC and ILC, arXiv:1404.7437 [INSPIRE].

[32] B. Dumont, J.F. Gunion, Y. Jiang and S. Kraml, Constraints on and future prospects for two-Higgs-doublet models in light of the LHC Higgs signal, Phys. Rev. D 90 (2014) 035021 [arXiv: 1405.3584] [INSPIRE].

[33] V. Ilisie and A. Pich, Low-mass fermiophobic charged Higgs phenomenology in two-Higgs-doublet models, JHEP 09 (2014) 089 [arXiv: 1405.6639] [INSPIRE].

[34] S. Kanemura, K. Tsumura, K. Yagyu and H. Yokoya, Fingerprinting non-minimal Higgs sectors, arXiv:1406.3294 [INSPIRE].

[35] A. Drozd, B. Grzadkowski and J. Wudka, Multi-scalar-singlet extension of the standard model - The case for dark matter and an invisible Higgs boson, JHEP 04 (2012) 006 [arXiv: 1112.2582] [INSPIRE].

[36] S. Bhattacharya, A. Drozd, B. Grzadkowski and J. Wudka, Two-component dark matter, JHEP 10 (2013) 158 [arXiv:1309.2986] [INSPIRE].

[37] X.-G. He, T. Li, X.-Q. Li, J. Tandean and H.-C. Tsai, Constraints on scalar dark matter from direct experimental searches, Phys. Rev. D 79 (2009) 023521 [arXiv:0811.0658] [INSPIRE].

[38] B. Grzadkowski and P. Osland, Tempered two-Higgs-doublet model, Phys. Rev. D 82 (2010) 125026 [arXiv: 0910.4068] [INSPIRE].

[39] M.S. Boucenna and S. Profumo, Direct and indirect singlet scalar dark matter detection in the lepton-specific two-Higgs-doublet model, Phys. Rev. D 84 (2011) 055011 [arXiv: 1106.3368] [INSPIRE].

[40] X.-G. He, B. Ren and J. Tandean, Hints of standard model Higgs boson at the LHC and light dark matter searches, Phys. Rev. D 85 (2012) 093019 [arXiv:1112.6364] [INSPIRE].

[41] Y. Bai, V. Barger, L.L. Everett and G. Shaughnessy, Two-Higgs-doublet-portal dark-matter model: LHC data and Fermi-LAT 135 GeV line, Phys. Rev. D 88 (2013) 015008 [arXiv: 1212.5604] [INSPIRE].

[42] X.-G. He and J. Tandean, Low-mass dark-matter hint from CDMS II, Higgs boson at the LHC and darkon models, Phys. Rev. D 88 (2013) 013020 [arXiv: 1304.6058] [INSPIRE]. 
[43] Y. Cai and T. Li, Singlet dark matter in a type-II two Higgs doublet model, Phys. Rev. D 88 (2013) 115004 [arXiv:1308.5346] [INSPIRE].

[44] L. Wang, A simplified 2HDM with a scalar dark matter and the galactic center gamma-ray excess, arXiv: 1406.3598 [INSPIRE].

[45] C.-Y. Chen, M. Freid and M. Sher, The next-to-minimal two Higgs doublet model, Phys. Rev. D 89 (2014) 075009 [arXiv: 1312.3949] [INSPIRE].

[46] J. Guo and Z. Kang, Higgs naturalness and dark matter stability by scale invariance, arXiv: 1401.5609 [INSPIRE].

[47] M. Kadastik, K. Kannike and M. Raidal, Matter parity as the origin of scalar dark matter, Phys. Rev. D 81 (2010) 015002 [arXiv: 0903.2475] [InSPIRE].

[48] M. Kadastik, K. Kannike and M. Raidal, Dark matter as the signal of grand unification, Phys. Rev. D 80 (2009) 085020 [Erratum ibid. D 81 (2010) 029903] [arXiv:0907.1894] [INSPIRE].

[49] M. Kadastik, K. Kannike, A. Racioppi and M. Raidal, EWSB from the soft portal into Dark Matter and prediction for direct detection, Phys. Rev. Lett. 104 (2010) 201301 [arXiv: 0912.2729] [INSPIRE].

[50] P.M. Ferreira, R. Santos, M. Sher and J.P. Silva, Implications of the LHC two-photon signal for two-Higgs-doublet models, Phys. Rev. D 85 (2012) 077703 [arXiv:1112.3277] [INSPIRE].

[51] P.M. Ferreira, R. Santos, M. Sher and J.P. Silva, Could the LHC two-photon signal correspond to the heavier scalar in two-Higgs-doublet models?, Phys. Rev. D 85 (2012) 035020 [arXiv: 1201.0019$]$ [INSPIRE].

[52] H.S. Cheon and S.K. Kang, Constraining parameter space in type-II two-Higgs doublet model in light of a $126 \mathrm{GeV}$ Higgs boson, JHEP 09 (2013) 085 [arXiv: 1207.1083] [INSPIRE].

[53] D.S.M. Alves, P.J. Fox and N.J. Weiner, Higgs signals in a type I 2HDM or with a sister Higgs, arXiv:1207.5499 [inSPIRE].

[54] W. Altmannshofer, S. Gori and G.D. Kribs, A minimal flavor violating 2HDM at the LHC, Phys. Rev. D 86 (2012) 115009 [arXiv:1210.2465] [InSPIRE].

[55] S. Chang et al., Comprehensive study of two Higgs doublet model in light of the new boson with mass around $125 \mathrm{GeV}$, JHEP 05 (2013) 075 [arXiv:1210.3439] [INSPIRE].

[56] P.M. Ferreira, R. Santos, H.E. Haber and J.P. Silva, Mass-degenerate Higgs bosons at $125 \mathrm{GeV}$ in the two-Higgs-doublet model, Phys. Rev. D 87 (2013) 055009 [arXiv:1211.3131] [INSPIRE].

[57] A. Drozd, B. Grzadkowski, J.F. Gunion and Y. Jiang, Two-Higgs-doublet models and enhanced rates for a $125 \mathrm{GeV}$ Higgs, JHEP 05 (2013) 072 [arXiv: 1211.3580] [INSPIRE].

[58] C.-Y. Chen and S. Dawson, Exploring two Higgs doublet models through Higgs production, Phys. Rev. D 87 (2013) 055016 [arXiv: 1301.0309] [inSPIRE].

[59] A. Celis, V. Ilisie and A. Pich, LHC constraints on two-Higgs doublet models, JHEP 07 (2013) 053 [arXiv:1302.4022] [INSPIRE].

[60] G. Bélanger, B. Dumont, U. Ellwanger, J.F. Gunion and S. Kraml, Global fit to Higgs signal strengths and couplings and implications for extended Higgs sectors, Phys. Rev. D 88 (2013) 075008 [arXiv: 1306 .2941] [INSPIRE]. 
[61] J.F. Gunion and H.E. Haber, The CP conserving two Higgs doublet model: The Approach to the decoupling limit, Phys. Rev. D 67 (2003) 075019 [hep-ph/0207010] [INSPIRE].

[62] J.F. Gunion, H.E. Haber, G.L. Kane, and S. Dawson, The Higgs hunter's guide, Front. Phys. 80 (2000) 1.

[63] G. Bélanger, B. Dumont, U. Ellwanger, J.F. Gunion and S. Kraml, Status of invisible Higgs decays, Phys. Lett. B 723 (2013) 340 [arXiv:1302.5694] [InSPIRE].

[64] J.R. Espinosa, M. Muhlleitner, C. Grojean and M. Trott, Probing for invisible Higgs decays with global fits, JHEP 09 (2012) 126 [arXiv:1205.6790] [INSPIRE].

[65] B. Grzadkowski and J. Wudka, Pragmatic approach to the little hierarchy problem: the case for Dark Matter and neutrino physics, Phys. Rev. Lett. 103 (2009) 091802 [arXiv: 0902.0628] [INSPIRE].

[66] R.A. Horn and C.R. Johnson, Matrix analysis, Cambridge University Press, Cambridge U.K. (1985).

[67] D. Eriksson, J. Rathsman and O. Stal, 2HDMC: two-Higgs-doublet model calculator physics and manual, Comput. Phys. Commun. 181 (2010) 189 [arXiv:0902.0851] [INSPIRE].

[68] D. Eriksson, J. Rathsman and O. Stal, 2HDMC: two-Higgs-doublet model calculator, Comput. Phys. Commun. 181 (2010) 833 [INSPIRE].

[69] E.W. Kolb and M.S. Turner, The Early universe, Front. Phys. 69 (1990) 1.

[70] Planck collaboration, P.A.R. Ade et al., Planck 2013 results. XVI. Cosmological parameters, Astron. Astrophys. (2014) [arXiv:1303.5076] [INSPIRE].

[71] G. Jungman, M. Kamionkowski and K. Griest, Supersymmetric dark matter, Phys. Rept. 267 (1996) 195 [hep-ph/9506380] [INSPIRE].

[72] G. Bélanger, F. Boudjema, A. Pukhov and A. Semenov, MicrOMEGAs_3: a program for calculating dark matter observables, Comput. Phys. Commun. 185 (2014) 960 [arXiv: 1305. 0237] [INSPIRE].

[73] CMS collaboration, Search for invisible decays of Higgs bosons in the vector boson fusion and associated ZH production modes, Eur. Phys. J. C 74 (2014) 2980 [arXiv:1404.1344] [INSPIRE].

[74] A.W. El Kaffas, W. Khater, O.M. Ogreid and P. Osland, Consistency of the two Higgs doublet model and CP-violation in top production at the LHC, Nucl. Phys. B 775 (2007) 45 [hep-ph/0605142] [INSPIRE].

[75] B. Grzadkowski, O.M. Ogreid and P. Osland, Natural multi-Higgs model with dark matter and CP-violation, Phys. Rev. D 80 (2009) 055013 [arXiv:0904.2173] [InSPIRE].

[76] A.G. Akeroyd, A. Arhrib and E.-M. Naimi, Note on tree level unitarity in the general two Higgs doublet model, Phys. Lett. B 490 (2000) 119 [hep-ph/0006035] [INSPIRE].

[77] A. Arhrib, Unitarity constraints on scalar parameters of the standard and two Higgs doublets model, hep-ph/0012353 [INSPIRE].

[78] J. Horejsi and M. Kladiva, Tree-unitarity bounds for THDM Higgs masses revisited, Eur. Phys. J. C 46 (2006) 81 [hep-ph/0510154] [inSPIRE].

[79] I.F. Ginzburg and I.P. Ivanov, Tree-level unitarity constraints in the most general 2HDM, Phys. Rev. D 72 (2005) 115010 [hep-ph/0508020] [InSPIRE]. 